\title{
BALANCED METRICS AND CHOW STABILITY OF PROJECTIVE BUNDLES OVER KÄHLER MANIFOLDS
} II

\author{
REZA SEYYEDALI
}

\begin{abstract}
In the previous article $([\underline{S}])$, we proved that slope stability of a holomorphic vector bundle $E$ over a polarized manifold $(X, L)$ implies Chow stability of $\left(\mathbb{P} E^{*}, \mathcal{O}_{\mathbb{P} E^{*}}(1) \otimes \pi^{*} L^{k}\right)$ for $k \gg 0$ if the base manifold has no nontrivial holomorphic vector field and admits a constant scalar curvature metric in the class of $2 \pi c_{1}(L)$. In this article using asymptotic expansions of Bergman kernel on $\operatorname{Sym}^{d} E$, we generalize the main theorem of $[\mathrm{S}]$ to polarizations $\left(\mathbb{P} E^{*}, \mathcal{O}_{\mathbb{P} E^{*}}(d) \otimes \pi^{*} L^{k}\right)$ for $k \gg 0$, where $d$ is a positive integer.
\end{abstract}

\section{INTRODUCTION}

In $[\mathbf{S}$, we prove that if a holomorphic vector bundle $E$ over a polarized algebraic manifold $(X, L)$ is Mumford stable and if $(X, L)$ admits a constant scalar curvature metric and has discrete automorphism group, then $\left(\mathbb{P} E^{*}, \mathcal{O}_{\mathbb{P} E^{*}}(1) \otimes \pi^{*} L^{k}\right)$ is Chow stable for $k \gg 0$. The goal of this article is to generalize this result for the polarizations $\left(\mathbb{P} E^{*}, \mathcal{O}_{\mathbb{P} E^{*}}(d) \otimes \pi^{*} L^{k}\right)$ for positive integer $d$ and $k \gg 0$. More precisely,

Theorem 1.1. Suppose that $A u t(X)$ is discrete and $X$ admits a constant scalar curvature Kähler metric in the class of $2 \pi c_{1}(L)$. Let $d$ be a positive integer. If $E$ is Mumford stable, then

$$
\left(\mathbb{P} E^{*}, \mathcal{O}_{\mathbb{P} E^{*}}(d) \otimes \pi^{*} L^{k}\right)
$$

is Chow stable for $k \gg 0$.

The proof of the Theorem for general $d$ as opposed to $d=1$ needs a new result for the asymptotic expansion of the Bergman kernel.

In order to prove Thm. 1.1 we use the concept of balanced metrics. Combining the results of Luo, Phong, Sturm and Zhang ([L], [PS1], [Zh]) on the relation between balanced metrics and stability, it suffices to prove the following

Date: April 20, 2011. 
Theorem 1.2. Let $X$ be a compact complex manifold and $L \rightarrow X$ be an ample line bundle. Suppose that $X$ admits a constant scalar curvature Kähler metric in the class of $2 \pi c_{1}(L)$ and $A u t(X)$ is discrete. Let $E \rightarrow X$ be a holomorphic vector bundle on $X$. If $E$ is Mumford stable, then $\mathcal{O}_{\mathbb{P} E^{*}}(d) \otimes \pi^{*} L^{k}$ admits balanced metrics for $k \gg 0$.

The balanced condition may be formulated in terms of Bergman kernels. First, we show that there exists an asymptotic expansion for the Bergman kernel of $\left(\mathbb{P} E^{*}, \mathcal{O}_{\mathbb{P} E^{*}}(d) \otimes \pi^{*} L^{k}\right)$. Fix a Kähler form $\omega$ on $X$ and a positive hermitian metric $\sigma$ on $L$ such that $i \bar{\partial} \partial \log \sigma=\omega$. For any positive hermitian metric $g$ on $\mathcal{O}_{\mathbb{P} E^{*}}(d)$, we define the sequence of volume forms $d \mu_{g, k}$ on $\mathbb{P} E^{*}$ as follows

$$
d \mu_{g, k}=k^{-m} \frac{\left(\omega_{g}+k \pi^{*} \omega\right)^{m+r-1}}{(m+r-1) !}=\sum_{j=0}^{m} k^{j-m} \frac{\omega_{g}^{m+r-1-j}}{(m+r-j) !} \wedge \frac{\pi^{*} \omega^{j}}{j !}
$$

where $\omega_{g}=i \bar{\partial} \partial \log g$.

Let $\rho_{k}(g, \omega)$ be the Bergman kernel of $H^{0}\left(\mathbb{P} E^{*}, \mathcal{O}_{\mathbb{P} E^{*}}(d) \otimes \pi^{*} L^{k}\right)$ with respect to the $L^{2}$-inner product $L^{2}\left(g \otimes \sigma^{\otimes k}, d \mu_{k, g}\right)$. We prove the following theorem.

Theorem 1.3. For any hermitian metric $h$ on $E$ and Kähler form $\omega \in 2 \pi c_{1}(L)$, there exist smooth endomorphisms $\widetilde{B}_{k}(h, \omega)$ such that

$$
\rho_{k}(g, \omega)([v])=C_{r, d}^{-1} \operatorname{tr}\left(\lambda_{d}\left(v, \operatorname{Sym}^{d} h\right) \widetilde{B}_{k}(h, \omega)\right),
$$

where $g$ is the Fubini-Study metric on $\mathcal{O}_{\mathbb{P} E^{*}}(d)$ induced by the hermitian metric $h, C_{r, d}$ is a constant defined by (2.1) and $\lambda_{d}\left(v, S^{2} m^{d} h\right)$ is an endomorphism of Sym $^{d} E$ defined in Def. 2.1. Moreover,

(1) There exist smooth endomorphisms $A_{i}(h, \omega) \in \Gamma\left(X, \operatorname{End}\left(\operatorname{Sym}^{d} E\right)\right)$ such that the following asymptotic expansion holds as $k \longrightarrow \infty$,

$$
\widetilde{B}_{k}(h, \omega) \sim k^{m}+A_{1}(h, \omega) k^{m-1}+\ldots .
$$

(2) In particular

$$
A_{1}(h, \omega)=\frac{r}{(r+d)}\left(\Lambda F_{S_{y m}^{d} h}-\frac{1}{R} \operatorname{tr}\left(\Lambda F_{S_{S y m}^{d} h}\right) I_{S^{\prime} m^{d} E}\right)+\frac{1}{2} S(\omega) I_{S y m^{d} E},
$$

where $\Lambda$ is the trace operator acting on $(1,1)$-forms with respect to the Kähler form $\omega, F_{S y m}^{d} h$ is the curvature of $\left(S^{d} m^{d} E, S^{d}{ }^{d} h\right)$, $R$ is the rank of the bundle $S_{y m}^{d} E$ and $S(\omega)$ is the scalar curvature of $\omega$.

(3) The asymptotic expansion holds in $C^{\infty}$. More precisely, for any positive integers $a$ and $p$, there exists a positive constant $K_{a, p, \omega, h}$ 
such that

$$
\left\|\widetilde{B}_{k}(h, \omega)-\left(k^{m}+\cdots+A_{p}(h, \omega) k^{m-p}\right)\right\|_{C^{a}} \leq K_{a, p, \omega, h} k^{m-p-1} .
$$

Moreover the expansion is uniform in the sense that there exists a positive integer s such that if $h$ and $\omega$ run in a bounded family in $C^{s}$ topology and $\omega$ is bounded from below, then the constants $K_{a, p, \omega, h}$ are bounded by a constant depending only on a and $p$.

Finding balanced metrics on $\mathcal{O}_{\mathbb{P} E^{*}}(d) \otimes \pi^{*} L^{k}$ is basically the same as finding solutions to the equations $\rho_{k}(g, \omega)=$ Constant. Therefore in order to prove Thm. 1.2, we need to solve the equations $\rho_{k}(g, \omega)=$ Constant for $k \gg 0$. Now if $h$ satisfies the Hermitian-Einstein equation $\Lambda_{\omega} F_{(E, h)}=\mu I_{E}$, then $\mathrm{Sym}^{d} h$ satisfies a Hermitian-Einstein equation as well. Therefore if $\omega$ has constant scalar curvature and $h$ satisfies the Hermitian-Einstein equation, then $A_{1}(h, \omega)$ is constant. Notice that in order to make $A_{1}$ constant, existence of Hermitian-Einstein metric is not enough. We need the existence of constant scalar curvature Kähler metric as well. Therefore if we know that the linearization of $A_{1}$ at $\left(h_{H E}, \omega_{C S K}\right)$ is surjective, we could construct formal solutions as power series in $k^{-1}$ for the equation $\rho_{k}(g, \omega)=$ Constant. Unfortunately the linearization of $A_{1}$ at $\left(h_{H E}, \omega_{C S K}\right)$ is only onto the subspace of hermitian endomorphisms of $\mathrm{Sym}^{d} E$ that are induced from endomorphisms of $E$. In order to overcome this issue, we generalize Theorem 1.3 to metrics of the form $\operatorname{Sym}^{d} h\left(I+k^{-1} \Phi\right)$, where $h$ is a metric on $E$ and $\Phi$ is a hermitian endomorphism of $\operatorname{Sym}^{d} E$. Let $g$ and $g_{k}(\Phi)$ be the Fubini-Study metrics on $\mathcal{O}_{\mathbb{P} E^{*}}(d)$ induced by $\mathrm{Sym}^{d} h$ and $\operatorname{Sym}^{d} h\left(I+k^{-1} \Phi\right)$ respectively. Let $\rho_{k}(g, \omega, \Phi)$ be the Bergman kernel of $\left(\mathcal{O}_{\mathbb{P} E^{*}}(d) \otimes \pi^{*} L^{k}\right)$ with respect to $L^{2}\left(g_{k}(\Phi) \otimes \sigma^{\otimes k}, d \mu_{k, g, \Phi}\right)$, where $d \mu_{k, g, \Phi}=k^{-m} \frac{\left(\omega_{g_{k}(\Phi)}+k \omega\right)^{m+r-1}}{(m+r-1) !}$ and $\omega_{g_{k}(\Phi)}=i \bar{\partial} \partial \log g_{k}(\Phi)$.

Theorem 1.4. Let $h$ be a hermitian metric on $E, \Phi$ be a hermitian endomorphism of $\operatorname{Sym}^{d} E$ and $\omega \in 2 \pi c_{1}(L)$ be a Kähler form. Then there exist smooth endomorphisms $\widetilde{B}_{k}(h, \omega, \Phi)$ such that

$$
\rho_{k}(g, \omega, \Phi)([v])=C_{r, d}^{-1} \operatorname{tr}\left(\lambda_{d}\left(v, \operatorname{Sym}^{d} h\left(I+k^{-1} \Phi\right)\right) \widetilde{B}_{k}(h, \omega, \Phi)\right),
$$

where $g$ is the Fubini-Study metric on $\mathcal{O}_{\mathbb{P} E^{*}}(d)$ induced by the hermitian metric h. Moreover,

(1) There exist smooth endomorphisms $A_{i}(h, \omega . \Phi) \in \Gamma\left(X, \operatorname{End}\left(\operatorname{Sym}^{d} E\right)\right)$ such that the following asymptotic expansion holds as $k \longrightarrow \infty$,

$$
\widetilde{B}_{k}(h, \omega, \Phi) \sim k^{m}+A_{1}(h, \omega, \Phi) k^{m-1}+\ldots
$$


(2) In particular

$$
A_{1}(h, \omega, \Phi)=A_{1}(h, \omega)+\Phi-T(\Phi)
$$

where $A_{1}(h, \omega)$ is given in Theorem 1.3 and $T: \operatorname{End}\left(\operatorname{Sym}^{d} E\right) \rightarrow$ $\operatorname{End}\left(\operatorname{Sym}^{d} E\right)$ is a bundle map defined in Def. 3.3.

(3) The asymptotic expansion holds in $C^{\infty}$. More precisely, for any positive integers a and $p$, there exists a positive constant $K_{a, p, \omega, h}$ such that

$$
|| \widetilde{B}_{k}(h, \omega, \Phi)-\left.\left(k^{m}+\cdots+A_{p}(h, \omega, \Phi) k^{m-p}\right)\right|_{C^{a}} \leq K_{a, p, \omega, h, \Phi} k^{m-p-1} .
$$

Moreover the expansion is uniform in the sense that there exists a positive integer $s$ such that if $h, \omega$ and $\Phi$ run in a bounded family in $C^{s}$ topology and $\omega$ and $h$ are bounded from below, then the constants $K_{a, p, \omega, h, \Phi}$ are bounded by a constant depending only on $a$ and $p$.

Next, the crucial fact is that the linearization of $A_{1}$ at $\left(h, \omega, I_{\mathrm{Sym}^{d} E}\right)$ is surjective. This enables us to construct formal solutions as power series in $k^{-1}$ for the equation $\rho_{k}(g, \omega)=$ Constant. Therefore, for any positive integer $q$, we can construct a sequence of metrics $g_{k}$ on $\mathcal{O}_{\mathbb{P} E^{*}}(d) \otimes \pi^{*} L^{k}$ and bases $s_{1}^{(k)}, \ldots, s_{N}^{(k)}$ for $H^{0}\left(\mathbb{P} E^{*}, \mathcal{O}_{\mathbb{P} E^{*}}(d)\right)$ such that

$$
\sum\left|s_{i}^{(k)}\right|_{g_{k}}^{2}=1, \text { and } \int\left\langle s_{i}^{(k)}, s_{j}^{(k)}\right\rangle_{g_{k}} d v o l_{g_{k}}=D_{k} I+M_{k},
$$

where $D_{k} \rightarrow C_{r, d}$ as $k \rightarrow \infty$ (See (2.1) for definition of $C_{r, d}$.), and $M_{k}$ is a trace-free hermitian matrix such that $\left\|M_{k}\right\|_{\text {op }}=o\left(k^{-q-1}\right)$ as $k \rightarrow \infty$. Now [S, Theorem 4.6.] implies that we can perturb these almost balanced metrics to get balanced metrics.

This article covers the following. In section 2, we review some basics about symmetric powers. In section 3, we prove the existence of an asymptotic expansions of Bergman kernels for $\left(\mathbb{P} E^{*}, \mathcal{O}_{\mathbb{P} E^{*}}(d) \otimes \pi^{*} L^{k}\right)$. We prove Theorem 1.3 and Theorem 1.4 in this section. Section 4 is devoted to construction of sequences of almost balanced metrics on $\left(\mathbb{P} E^{*}, \mathcal{O}_{\mathbb{P} E^{*}}(d) \otimes \pi^{*} L^{k}\right)$ using Theorem 1.4. In section 5, we prove Theorem 3.1 and Theorem 3.7 which guarantee that the asymptotic expansions obtained in Theorem 1.3 and Theorem 1.4 hold in $C^{\infty}$.

We refer the reader to [S] for a history on the subject and related results.

Acknowledgements: I am sincerely grateful to Richard Wentworth for many helpful discussions and suggestions and his continuous help, support and encouragement. I would also like to thank Zhiqin Lu for many helpful discussions and suggestions. 


\section{Preliminaries}

Let $V$ be a complex vector space of dimension $r$. We define

$$
\operatorname{Sym}^{d} V=V^{\otimes d} / \sim
$$

where

$$
v_{1} \otimes \cdots \otimes v_{d} \sim v_{\sigma(1)} \otimes \cdots \otimes v_{\sigma(d)}
$$

for any $\sigma \in S_{d}$. We simply denote the class of $v_{1} \otimes \cdots \otimes v_{d}$ in $\operatorname{Sym}^{d} V$ by $v_{1} \ldots v_{d}$.

Any hermitian inner product $h$ on $V$ defines a hermitian inner product $\mathrm{Sym}^{d} h$ on $\operatorname{Sym}^{d} V$ by

$$
<v_{1} \ldots v_{d}, w_{1} \ldots w_{d}>_{\mathrm{Sym}^{d} h}=\frac{1}{d !} \sum_{\sigma \in S_{d}}<v_{1}, w_{\sigma(1)}>\cdots<v_{d}, w_{\sigma(d)}>\text {. }
$$

Lemma 2.1. Let $e_{1}, \ldots, e_{r}$ be a basis for $V$, then

$$
\left\{e_{1}^{i_{1}} \ldots e_{r}^{i_{r}} \mid 0 \leq i_{\alpha} \leq d, \sum_{\alpha=1}^{r} j_{\alpha}=d\right\}
$$

forms a basis for $S_{y m}^{d} V$. Moreover, if the basis $e_{1}, \ldots, e_{r}$ is an orthonormal basis with respect to $h$, then

$$
\begin{gathered}
<e_{1}^{i_{1}} \ldots e_{r}^{i_{r}}, e_{1}^{j_{1}} \ldots e_{r}^{i_{r}}>_{S y m^{d} h}=0 \quad \text { if }\left(i_{1}, \ldots, i_{r}\right) \neq\left(j_{1}, \ldots, j_{r}\right), \\
\left\|e_{1}^{i_{1}} \ldots e_{r}^{i_{r}}\right\|_{S y m^{d} h}^{2}=\frac{i_{1} ! \ldots i_{r} !}{d !}
\end{gathered}
$$

where $i_{1}, \ldots, i_{r}, j_{1}, \ldots, j_{r}$ are integers such that $0 \leq i_{\alpha}, j_{\alpha} \leq d$ and $\sum_{\alpha=1}^{r} i_{\alpha}=\sum_{\alpha=1}^{r} j_{\alpha}=d$.

Definition 2.1. For any hermitian inner product $H$ on $\operatorname{Sym}^{d} V$ and $v \in$ $V$, define an endomorphism $\lambda_{d}(v, H)$ of $\operatorname{Sym}^{d} V$ by

$$
\lambda_{d}(v, H)=\left|v_{d}\right|_{H}^{-2} v_{d} \otimes v_{d}^{*_{H}},
$$

where $v_{d}=v \ldots v$. For any hermitian inner product $H$ on $\operatorname{Sym}^{d} V$ and $v^{*} \in V^{*}$, define an endomorphism $\lambda_{d}\left(v^{*}, H\right)$ of $\operatorname{Sym}^{d} V$ by

$$
\lambda_{d}\left(v^{*}, H\right)=|w|_{H}^{-2} w \otimes w^{* H},
$$

where $w$ is the unique vector in $\operatorname{Sym}^{d} V$ satisfying

$$
v_{d}^{*}(u)=\langle u, w\rangle_{H} \quad \forall u \in \operatorname{Sym}^{d} V .
$$

There is a natural isomorphism between $\operatorname{Sym}^{d} V$ and $H^{0}\left(\mathbb{P} V^{*}, \mathcal{O}_{\mathbb{P} V^{*}}(d)\right)$ which sends $v_{1} \ldots v_{d} \in \operatorname{Sym}^{d} V$ to ${\widehat{v_{1} \ldots v_{d}}}_{d} \in H^{0}\left(\mathbb{P} V^{*}, \mathcal{O}_{\mathbb{P} V^{*}}(d)\right)$ defined by

$$
{\widehat{v_{1} \ldots v_{d}}}_{d}\left(\left[v^{*}\right]\right)\left(w_{1}^{*} \otimes \cdots \otimes w_{d}^{*}\right)=w_{1}^{*}\left(v_{1}\right) \ldots w_{d}^{*}\left(v_{d}\right)
$$


where $w_{1}^{*} \in V^{*}$ and there exist complex numbers $\lambda_{1}, \ldots, \lambda_{d}$ such that $w_{i}^{*}=\lambda_{i} v^{*}$. Notice that

$$
{\widehat{v_{1} \ldots v_{d}}}_{d}\left(\left[v^{*}\right]\right)\left(w_{1}^{*} \otimes \cdots \otimes w_{d}^{*}\right)=\lambda_{1} \ldots \lambda_{d} v^{*}\left(v_{1}\right) \ldots v^{*}\left(v_{d}\right)
$$

and therefore it is a well-defined section of $\mathcal{O}_{\mathbb{P} V^{*}}(d)$.

For any hermitian inner product $H$ on $\operatorname{Sym}^{d} V$, define a metric $\widehat{H}$ on $\mathcal{O}_{\mathbb{P} V^{*}}(d)$ by

$$
\langle\hat{s}, \hat{t}\rangle_{\widehat{H}}[v]=\frac{v^{\otimes d}(s) \overline{v^{\otimes d}(t)}}{|v \ldots v|_{H}^{2}} .
$$

Note that originally $H$ is a hermitian inner product on $\operatorname{Sym}^{d} V$ and $v \ldots v \in \operatorname{Sym}^{d} V^{*}$. However, the hermitian inner product $H$ induces a hermitian inner product on $\mathrm{Sym}^{d} V^{*}$ which we denote it by $H$ as well. In particular

$$
\langle\hat{s}, \hat{t}\rangle_{\widehat{\operatorname{Sym}^{d} h}}[v]=\frac{v^{\otimes d}(s) \overline{v^{\otimes d}(t)}}{|v|_{h}^{2 d}} .
$$

The following lemma is straightforward.

Lemma 2.2. For any hermitian inner product $h$ on $V$, we have

$$
\hat{h}^{\otimes d}=\widehat{S y m^{d} h} .
$$

Lemma 2.3. There exists a constant $C_{r, d}$ such that for any $v, w \in$ $\operatorname{Sym}^{d} V$ and any hermitian inner product $h$ on $V$,

$$
d^{r-1} \int_{\mathbb{P} V^{*}}\langle\hat{v}, \hat{w}\rangle_{\widehat{h} \otimes d} \frac{\omega_{\hat{h}}^{r-1}}{(r-1) !}=C_{r, d}\langle v, w\rangle_{S y m^{d} h},
$$

where $\omega_{\hat{h}}=i \bar{\partial} \partial \log \hat{h}$. The constant $C_{r, d}$ is given by the following formula

$$
C_{r, d}=\int_{\mathbb{C}^{r-1}} \frac{d \xi \wedge d \bar{\xi}}{\left(1+\sum_{j=1}^{r-1}\left|\xi_{j}\right|^{2}\right)^{r+d}} .
$$

Here $d \xi \wedge d \bar{\xi}=\left(\sqrt{-1} d \xi_{1} \wedge d \bar{\xi}_{1}\right) \wedge \cdots \wedge\left(\sqrt{-1} d \xi_{r-1} \wedge d \bar{\xi}_{r-1}\right)$.

Conversely, let $H$ be a hermitian inner product on $S_{y m}{ }^{d} V$. Suppose there exists a constant $C$ such that

$$
\int_{\mathbb{P} V^{*}}\langle\hat{v}, \hat{w}\rangle_{\widehat{H}} \frac{\omega_{\widehat{H}}^{r-1}}{(r-1) !}=C\langle v, w\rangle_{H},
$$

for any $v, w \in S y m^{d} V$. Then there exists a hermitian inner product $h$ on $V$ such that $H=S_{y m}^{d} h$. 
Proof. The first part is a straightforward computation. For the second part, suppose that $H$ is a hermitian inner product on $\operatorname{Sym}^{d} V$ satisfying

$$
\int_{\mathbb{P} V^{*}}\langle\hat{v}, \hat{w}\rangle_{\widehat{H}} \frac{\omega_{\widehat{H}}^{r-1}}{(r-1) !}=C\langle v, w\rangle_{H},
$$

for any $v, w \in \operatorname{Sym}^{d} V$. Let $v_{1}, \ldots v_{R}$ be an orthonormal basis for $\operatorname{Sym}^{d} V$ with respect to $H$. For any $e^{*} \in V^{*}$, we have

$$
\sum\left\|\widehat{v}_{i}\right\|_{\widehat{H}}^{2}\left(\left[e^{*}\right]\right)=\frac{\sum e_{d}^{*}\left(v_{i}\right) \overline{e_{d}^{*}\left(v_{i}\right)}}{\left\|e_{d}^{*}\right\|_{H}^{2}}=\frac{\left\|e_{d}^{*}\right\|_{H}^{2}}{\left\|e_{d}^{*}\right\|_{H}^{2}}=1 .
$$

On the other hand

$$
\int_{\mathbb{P} V^{*}}\left\langle\hat{v}_{i}, \hat{v}_{j}\right\rangle_{\widehat{H}} \frac{\omega_{\widehat{H}}^{r-1}}{(r-1) !}=C\left\langle v_{i}, v_{j}\right\rangle_{H}=C \delta_{i j} .
$$

Therefore $\widehat{H}$ is a balanced metric on $\left(\mathbb{P} V^{*}, \mathcal{O}_{\mathbb{P} V^{*}}(d)\right)$. It concludes the proof since balanced metrics on $\left(\mathbb{P} V^{*}, \mathcal{O}_{\mathbb{P} V^{*}}(d)\right)$ are unique up to $\operatorname{Aut}\left(\mathbb{P} V^{*}, \mathcal{O}_{\mathbb{P} V^{*}}(d)\right) \cong P G L(V)$.

There is a canonical representation of $\mathrm{Sym}^{d}: G L(V) \rightarrow G L\left(\mathrm{Sym}^{d} V\right)$ defined as follows:

$$
\left(\operatorname{Sym}^{d} A\right)\left(v_{1} \ldots v_{d}\right)=A v_{1} \ldots A v_{d}
$$

where $A \in G L(V)$ and $v_{1}, \ldots, v_{d} \in V$. This induces a Lie algebra homomorphism $S^{d}: \operatorname{End}(V) \rightarrow \operatorname{End}\left(\mathrm{Sym}^{d} V\right)$ defined by

$$
\left(S^{d} A\right)\left(v_{1} \ldots v_{d}\right)=\sum_{i=1}^{d} v_{1} \ldots A v_{i} \ldots v_{d}
$$

for any $A \in \operatorname{End}(V)$. Suppose that the vector space $V$ is equipped with a hermitian inner product. Then the Lie algebra homomorphism $S^{d}$ maps hermitian endomorphisms to hermitian endomorphisms. More precisely, we have the following.

Lemma 2.4. Let $h$ be a hermitian inner product on $V$. We denote the space of hermitian endomorphisms of $V$ with respect to $h$ by $\operatorname{End}_{h}(V)$ and the space of hermitian endomorphisms of $\operatorname{Sym}^{d} V$ with respect to $\operatorname{Sym}^{d} h$ by $\operatorname{End}_{h}\left(\operatorname{Sym}^{d} V\right)$. Then

$$
S^{d}\left(\operatorname{End}_{h}(V)\right) \subset \operatorname{End}_{h}\left(\operatorname{Sym}^{d} V\right) .
$$

Let $E$ be a holomorphic vector bundle over a Kähler manifold $(X, \omega)$ and $h$ be a hermitian metric on $E$. Then straightforward computation shows that

$$
F_{\left(\bar{\partial}_{\mathrm{Sym}^{d} E}, \operatorname{Sym}^{d} h\right)}=S^{d} F_{\left(\bar{\partial}_{E}, h\right)},
$$


where $F_{\left(\bar{\partial}_{E}, h\right)}$ is the curvature of the chern connection on $(E, h)$. A direct consequence of the above formula is the following:

Proposition 2.5. Let $h_{H E}$ be a Hermitian-Einstein metric on E with respect to $\omega$, i.e. $\Lambda_{\omega} F_{h_{H E}}=\mu I_{E}$. Then Sym ${ }^{d} h_{H E}$ is a HermitianEinstein metric on $\operatorname{Sym}^{d} \mathrm{E}$ with respect to $\omega$.

\section{Asymptotic Expansion}

The goal of this section is to give an asymptotic expansion for the Bergman kernel of $\left(\mathbb{P} E^{*}, \mathcal{O}_{\mathbb{P} E^{*}}(d) \otimes \pi^{*} L^{k}\right)$.

Let $(X, \omega)$ be a Kähler manifold of dimension $m$ and $E$ be a holomorphic vector bundle on $X$ of rank $r$. Let $L$ be an ample line bundle on $X$ endowed with a hermitian metric $\sigma$ such that $i \bar{\partial} \partial \log \sigma=\omega$. For any hermitian metric $h$ on $E$, we define the volume form

$$
d \mu_{g}=\frac{\omega_{g}^{r-1}}{(r-1) !} \wedge \frac{\pi^{*} \omega^{m}}{m !}
$$

where $g=\widehat{\operatorname{Sym}^{d} h}=\widehat{h}^{\otimes d}, \omega_{g}=i \bar{\partial} \partial \log g=d i \bar{\partial} \partial \log \widehat{h}$ and $\pi: \mathbb{P} E^{*} \rightarrow X$ is the projection map. The goal is to find an asymptotic expansion for the Bergman kernel of $\mathcal{O}_{\mathbb{P} E^{*}}(d) \otimes L^{k} \rightarrow \mathbb{P} E^{*}$ with respect to the $L^{2}$ metric defined on $H^{0}\left(\mathbb{P} E^{*}, \mathcal{O}_{\mathbb{P} E^{*}}(d) \otimes \pi^{*} L^{k}\right)$. We define the $L^{2}$ - metric using the fibre metric $g \otimes \sigma^{\otimes k}$ and the volume form $d \mu_{g, k}$ defined as follows

$$
d \mu_{g, k}=k^{-m} \frac{\left(\omega_{g}+k \omega\right)^{m+r-1}}{(m+r-1) !}=\sum_{j=0}^{m} k^{j-m} \frac{\omega_{g}^{m+r-1-j}}{(m+r-j) !} \wedge \frac{\omega^{j}}{j !} .
$$

In order to do that, we reduce the problem to the problem of Bergman kernel asymptotics on $\mathrm{Sym}^{d} E \otimes L^{k} \rightarrow X$. The first step is to use the volume form $d \mu_{g}$ which is a product volume form instead of the more complicated one $d \mu_{g, k}$. So, we replace the volume form $d \mu_{g, k}$ with $d \mu_{g}$ and the fibre metric $g \otimes \sigma^{k}$ with $g(k) \otimes \sigma^{k}$, where the metrics $g(k)$ are defined on $\mathcal{O}_{\mathbb{P} E^{*}}(d)$ by

$$
g(k)=k^{-m}\left(\sum_{j=0}^{m} k^{j} f_{j}\right) g=\left(f_{m}+k^{-1} f_{m-1}+\ldots+k^{-m} f_{0}\right) g,
$$

and

$$
\frac{\omega_{g}^{m+r-1-j}}{(m+r-j) !} \wedge \frac{\omega^{j}}{j !}=f_{j} d \mu_{g}
$$

Clearly the $L^{2}$-inner products $L^{2}\left(g \otimes \sigma^{k}, d \mu_{g, k}\right)$ and $L^{2}\left(g(k) \otimes \sigma^{k}, d \mu_{g}\right)$ on $H^{0}\left(\mathbb{P} E^{*}, \mathcal{O}_{\mathbb{P} E^{*}}(d) \otimes \pi^{*} L^{k}\right)$ are the same. The second step is going from $\mathcal{O}_{\mathbb{P} E^{*}}(d) \rightarrow \mathbb{P} E^{*}$ to $\operatorname{Sym}^{d} E \rightarrow X$. In order to do this we somehow push 
forward the metric $g(k)$ to get a metric $\widetilde{g}(k)$ on $\operatorname{Sym}^{d} E$ (See Definition 3.2). Then we can apply the result on the asymptotics of the Bergman kernel on $\mathrm{Sym}^{d} E$. The last step is to use this to get the result.

Definition 3.1. Let $\widehat{s_{1}^{k}}, \ldots, \widehat{s_{N}^{k}}$ be an orthonormal basis for $H^{0}\left(\mathbb{P} E^{*}, \mathcal{O}_{\mathbb{P} E^{*}}(d) \otimes\right.$ $\left.\pi^{*} L^{k}\right)$ with respect to $L^{2}\left(g \otimes \sigma^{k}, d \mu_{k, g}\right)$. We define

$$
\rho_{k}(g, \omega)=\sum_{i=1}^{N}\left|\widehat{s_{i}^{k}}\right|_{g \otimes \sigma^{k}}^{2} .
$$

Definition 3.2. For any hermitian form $g$ on $\mathcal{O}_{\mathbb{P} E^{*}}(d)$, we define a hermitian form $\widetilde{g}$ on $\mathrm{Sym}^{d} E$ as follow

$$
\widetilde{g}(s, t)=C_{r, d}^{-1} \int_{\mathbb{P} E_{x}^{*}} g(\widehat{s}, \widehat{t}) \frac{\omega_{g}^{r-1}}{(r-1) !},
$$

for $s, t \in \operatorname{Sym}^{d} E_{x}$. (See (2.1) for definition of $C_{r, d}$.)

Notice that if $g=\widehat{\operatorname{Sym}^{d} h}$ for some hermitian metric $h$ on $E$, Lemma 2.3 implies that $\widetilde{g}=\operatorname{Sym}^{d} h$. Define hermitian metrics $\widetilde{g}_{j}$ 's on $\mathrm{Sym}^{d} E$ by

$$
\widetilde{g}_{j}(s, t)=C_{r, d}^{-1} \int_{\mathbb{P} E_{x}^{*}} f_{j} g(\widehat{s}, \widehat{t}) \frac{\omega_{g}^{r-1}}{(r-1) !},
$$

for $s, t \in \operatorname{Sym}^{d} E_{x}$. Also we define $\Psi_{j} \in E n d\left(\operatorname{Sym}^{d} E\right)$ by

$$
\widetilde{g_{j}}=\operatorname{Sym}^{d} h \Psi_{j} \text {. }
$$

If $h$ and $\omega$ vary in bounded family, then $\Psi_{j}$ 's vary in a bounded family. More precisely, we have the following

Theorem 3.1. Let $\nu_{0}$ be a fixed Kähler form on $X$ and $h_{0}$ be a fixed hermitian metric on $E$. For any positive numbers $l$ and $l^{\prime}$ and any positive integer $p$, there exists a positive number $C_{l, l^{\prime}, p}$ such that if

$$
\|\omega\|_{C^{p}\left(\nu_{0}\right)},\|h\|_{C^{p+2}\left(h_{0}, \nu_{0}\right)} \leq l \quad \text { and } \quad \inf _{x \in X}\left|\omega(x)^{m}\right|_{\nu_{0}(x)} \geq l^{\prime},
$$

then $\left\|\Psi_{i}\right\|_{C^{p}\left(h_{0}, \nu_{0}\right)} \leq C_{l, l^{\prime}, p}$, for any $1 \leq i \leq m$.

We prove Theorem 3.1 in Section 5.

Lemma 3.2. We have the following

(1) $\Psi_{m}=I_{\text {Sym }^{d} E}$.

(2) $\Psi_{m-1}=\frac{d}{(r+d)}\left(\Lambda F_{S m^{d} h}+\operatorname{tr}\left(\Lambda F_{h}\right) I_{S y m^{d} E}\right)$. 
Proof. Fix a point $p \in X$. Let $e_{1}, \ldots, e_{r}$ be a local holomorphic frame for $E$ around $p$ such that

$$
\left\langle e_{i}, e_{j}\right\rangle_{h}(p)=\delta_{i j}, \quad d\left\langle e_{i}, e_{j}\right\rangle_{h}(p)=0 .
$$

For simplicity, we assume that

$$
\frac{i}{2 \pi} F_{h}(p)=\left(\begin{array}{cccc}
\omega_{1} & 0 & \cdots & 0 \\
0 & \omega_{2} & \cdots & 0 \\
\vdots & & \ddots & \vdots \\
0 & 0 & \cdots & \omega_{r}
\end{array}\right) .
$$

Let $\lambda_{1}, \ldots, \lambda_{r}$ be the homogeneous coordinates on the fibre. At the fixed point $p$, we have

$$
\omega_{g}=d\left(\omega_{\mathrm{FS}, h}+\frac{\sum \omega_{i}\left|\lambda_{i}\right|^{2}}{\sum\left|\lambda_{i}\right|^{2}}\right) .
$$

Therefore,

$$
\omega_{g}^{r} \wedge \omega^{m-1}=d \omega_{\mathrm{FS}, h}^{r-1} \wedge\left(\frac{\sum \omega_{i}\left|\lambda_{i}\right|^{2}}{\sum\left|\lambda_{i}\right|^{2}}\right) \wedge \omega^{m-1} .
$$

Hence

$$
f_{m-1}=d \frac{\sum\left|\lambda_{i}\right|^{2} \Lambda \omega_{i}}{\sum\left|\lambda_{i}\right|^{2}}
$$

Let $\alpha_{1}, \ldots, \alpha_{r}$ be nonnegative integers such that $\alpha_{1}+\cdots+\alpha_{r}=d$. Therefore,

$$
\begin{aligned}
\widetilde{g_{m-1}}\left(e_{1}^{\alpha_{1}} \ldots e_{r}^{\alpha_{r}}, e_{1}^{\alpha_{1}} \ldots e_{r}^{\alpha_{r}}\right) & =C_{r, d}^{-1} \pi_{*}\left(f_{m-1} \tilde{g}\left(e_{1}^{\alpha_{1} \ldots e_{r}^{\alpha_{r}}}, e_{1}^{\alpha \alpha_{1} \ldots e_{r}^{\alpha_{r}}}\right) \frac{\omega_{g}^{r-1}}{(r-1) !}\right) \\
= & C_{r, d}^{-1} C_{r} \sum \Lambda \omega_{i} \int_{\mathbb{C}^{r-1}} \frac{\left|\lambda_{i}\right|^{2}\left|\lambda_{1}\right|^{2 \alpha_{1}} \ldots\left|\lambda_{r}\right|^{2 \alpha_{r}} d \lambda \wedge d \bar{\lambda}}{\left(1+\sum_{j=1}^{r-1}\left|\lambda_{j}\right|^{2}\right)^{r+d+1}} \\
= & C_{r, d}^{-1} C_{r} \frac{r ! \alpha_{1} ! \ldots \alpha_{r} !}{(r+d) !} \sum_{i=1}^{r}\left(\alpha_{i}+1\right) \Lambda \omega_{i} .
\end{aligned}
$$

Hence,

$$
\Psi_{m-1}=\frac{d}{(r+d)}\left(\Lambda F_{S y m^{d} h}+\operatorname{tr}\left(\Lambda F_{h}\right) I_{S y m^{d} E}\right)
$$

The following lemmas are straightforward.

Lemma 3.3. $\widetilde{g \otimes \sigma^{k}}=\widetilde{g} \otimes \sigma^{k}$. 
Lemma 3.4. Let $H$ be a hermitian metric on $S y m^{d} E$ and $s_{1}, \ldots, s_{N}$ be a basis for $H^{0}\left(X, S^{d} m^{d} E\right)$. Then

$$
\sum\left|\widehat{s}_{i}\left(\left[v^{*}\right]\right)\right|_{\widehat{H}}^{2}=\operatorname{Tr}\left(B \lambda_{d}\left(v^{*}, H\right)\right),
$$

where $B=\sum s_{i} \otimes s_{i}^{*_{H}}$.

Proof of Theorem 1.3. Define

$$
h(k)=C_{r, d}^{-1} \pi_{*}\left(g \frac{d \mu_{g, k}}{d \mu_{g}} \frac{\omega_{g}^{r-1}}{(r-1) !}\right),
$$

i.e. for any $x \in X$ and $s, t \in \operatorname{Sym}^{d} E_{x}$, we have

$$
\langle s, t\rangle_{h(k)}=C_{r, d}^{-1} \int_{\mathbb{P} E_{x}^{*}}\langle\widehat{s}, \widehat{t}\rangle_{g} \frac{d \mu_{g, k}}{d \mu_{g}} \frac{\omega_{g}^{r-1}}{(r-1) !} .
$$

Therefore (3.1), (3.3) and (3.7) imply that

$$
h(k)=\sum_{j=0}^{m} k^{j-m} \widetilde{g}_{j}=\operatorname{Sym}{ }^{d} h\left(\sum_{j=0}^{m} k^{j-m} \Psi_{j}\right) .
$$

Let $B_{k}(h(k), \omega)$ be the Bergman kernel of $\operatorname{Sym}^{d} E \otimes L^{k}$ with respect to the $L^{2}$-metric defined by the hermitian metric $h(k) \otimes \sigma^{k}$ on $\operatorname{Sym}^{d} E \otimes L^{k}$ and the volume form $\frac{\omega^{m}}{m !}$ on $X$. Therefore, if $s_{1}, \ldots, s_{N}$ is an orthonormal basis for $H^{0}\left(X, \operatorname{Sym}^{d} E \otimes L^{k}\right)$ with respect to the $L^{2}\left(h(k) \otimes \sigma^{k}, \frac{\omega^{m}}{m !}\right)$, then

$$
B_{k}(h(k), \omega)=\sum s_{i} \otimes s_{i}^{* h(k) \otimes \sigma^{k}},
$$

We define $\widetilde{B}_{k}(h, \omega)$ as follows:

$$
\widetilde{B}_{k}(h, \omega)=\sum s_{i} \otimes s_{i}^{{ }^{*}{ }^{S y m} d h \otimes \sigma k} .
$$

Let $\widehat{s_{1}}, \ldots, \widehat{s_{N}}$ be the corresponding basis for $H^{0}\left(\mathbb{P} E^{*}, \mathcal{O}_{\mathbb{P} E^{*}}(d) \otimes L^{k}\right)$. Hence,

$$
\begin{aligned}
\int_{\mathbb{P} E^{*}}\left\langle\widehat{s_{i}}, \widehat{s_{j}}\right\rangle_{g \otimes \sigma^{k}} d \mu_{g, k} & =\int_{\mathbb{P} E^{*}}\left\langle\widehat{s_{i}}, \widehat{s_{j}}\right\rangle_{g \otimes \sigma^{k}}\left(\sum_{j=0}^{m} k^{j} f_{j}\right) d \mu_{g} \\
& =\int_{\mathbb{P} E^{*}}\left\langle\widehat{s_{i}}, \widehat{s_{j}}\right\rangle_{g(k) \otimes \sigma^{k}} d \mu_{g} \\
& =C_{r, d} \int_{X}\left\langle s_{i}, s_{j}\right\rangle_{h(k) \otimes \sigma^{k}} \frac{\omega^{m}}{m !} \\
& =C_{r, d} \delta_{i j} .
\end{aligned}
$$


Therefore $\frac{1}{\sqrt{C_{r, d}}} \widehat{s_{1}}, \ldots, \frac{1}{\sqrt{C_{r, d}}} \widehat{s_{N}}$ is an orthonormal basis for $H^{0}\left(\mathbb{P} E^{*}, \mathcal{O}_{\mathbb{P} E^{*}}(d) \otimes\right.$ $\left.L^{k}\right)$ with respect to $L^{2}\left(g \otimes \sigma^{k}, d \mu_{k, g}\right)$. Hence Lemma 3.4 implies that

$$
C_{r, d} \rho_{k}(g, \omega)=\operatorname{Tr}\left(\lambda_{d}\left(v^{*}, \operatorname{Sym}^{d} h\right) \widetilde{B}_{k}(h, \omega)\right) .
$$

Now, in order to conclude the proof, it suffices to show that there exist smooth endomorphisms $A_{i} \in \Gamma\left(X, \operatorname{End}\left(\operatorname{Sym}^{d} E\right)\right)$ such that

$$
\widetilde{B}_{k}(h, \omega) \sim k^{m}+A_{1} k^{m-1}+\ldots .
$$

Let $B_{k}\left(\mathrm{Sym}^{d} h, \omega\right)$ be the Bergman kernel of $\mathrm{Sym}^{d} E \otimes L^{k}$ with respect to the $L^{2}\left(\operatorname{Sym}^{d} h \otimes \sigma^{k}, \frac{\omega^{m}}{m !}\right)$. A fundamental result on the asymptotics of the Bergman kernel $([\mathrm{C}],[\mathrm{Z},[\mathrm{Lu}],[\mathrm{W}])$ states that there exists an asymptotic expansion

$$
B_{k}\left(\operatorname{Sym}^{d} h, \omega\right) \sim k^{m}+B_{1}\left(\operatorname{Sym}^{d} h\right) k^{m-1}+\ldots,
$$

where

$$
B_{1}\left(\operatorname{Sym}^{d} h\right)=\frac{i}{2 \pi} \Lambda F_{\left(\operatorname{Sym}^{d} E, \operatorname{Sym}^{d} h\right)}+\frac{1}{2} S(\omega) I_{\mathrm{Sym}^{d} E} \cdot
$$

(See also [BBS.) Moreover this expansion holds uniformly for any $h$ in a bounded family. Therefore, we can Taylor expand the coefficients $B_{i}\left(\operatorname{Sym}^{d} h\right)$ 's. We conclude that for endomorphisms $\Phi_{1}, \ldots, \Phi_{M}$,

$$
B_{k}\left(\operatorname{Sym}^{d} h\left(I+\sum_{i=0}^{M} k^{-i} \Phi_{i}\right), \omega\right) \sim k^{m}+B_{1}\left(\operatorname{Sym}^{d} h\right) k^{m-1}+\ldots
$$

Note that $B_{1}\left(\mathrm{Sym}^{d} h\right)$ in the above expansion does not depend on $\Phi_{i}$ 's and is given as before by

$$
B_{1}\left(\operatorname{Sym}^{d} h\right)=\frac{i}{2 \pi} \Lambda F_{\left(\operatorname{Sym}^{d} E, \operatorname{Sym}^{d} h\right)}+\frac{1}{2} S(\omega) I_{\mathrm{Sym}^{d} E} \cdot
$$

On the other hand

$$
\begin{aligned}
B_{k}(h(k), \omega)=\sum s_{i} \otimes s_{i}^{* \widetilde{g(k)} \otimes \sigma^{k}} & =\left(\sum s_{i} \otimes s_{i}^{{ }^{h}{ }^{\otimes \sigma} k}\right)\left(\sum_{j=0}^{m} k^{j-m} \Psi_{j}\right) \\
& =\widetilde{B}_{k}(h, \omega)\left(\sum_{j=0}^{m} k^{j-m} \Psi_{j}\right) .
\end{aligned}
$$

Therefore,

$$
\begin{aligned}
\widetilde{B}_{k}\left(\operatorname{Sym}^{d} h, \omega\right) & =B_{k}(h(k), \omega)\left(\sum_{j=0}^{m} k^{j-m} \Psi_{j}\right)^{-1} \\
& \sim k^{m}+\left(B_{1}\left(\operatorname{Sym}^{d} h\right)-\Psi_{m-1}\right) k^{m-1}+\ldots
\end{aligned}
$$


We have

$$
\begin{aligned}
B_{1}-\Psi_{m-1} & =\frac{i r}{2 \pi(r+d)} \Lambda F_{\mathrm{Sym}^{d} h}+\frac{1}{2} S(\omega) I_{\mathrm{Sym}^{d} E}-\frac{i d}{2 \pi(r+d)} \operatorname{tr}\left(\Lambda F_{h}\right) I_{S^{\prime} m^{d} E} \\
& =\frac{i r}{2 \pi(r+d)}\left(\Lambda F_{\mathrm{Sym}^{d} h}-\frac{1}{\operatorname{rank}\left(\operatorname{Sym}^{d} E\right)} \operatorname{tr}\left(\Lambda F_{\mathrm{Sym}^{d} h}\right) I\right)+\frac{1}{2} S(\omega) I
\end{aligned}
$$

Notice that Theorem 3.1 implies that if $h$ and $\omega$ vary in a bounded family and $\omega$ is bounded from below, then $\Psi_{1}, . ., \Psi_{m}$ vary in a bounded family. Therefore the asymptotic expansion that we obtained for $\widetilde{B}_{k}(h, \omega)$ is uniform as long as $h$ and $\omega$ vary in a bounded family and $\omega$ is bounded from below.

Suppose that $E$ admits a Hermitian-Einstein metric $h_{H E}$ and $(X, L)$ admits a constant scalar curvature Kähler metric $\omega_{C S C K}$. If the linearization of $A_{1}$ at $\left(h_{H E}, \omega_{C S C K}\right)$ were surjective, then we would be able to construct sequences of almost balanced metrics. The problem is that the image of the linearization of $A_{1}$ consists only those endomorphisms of $\operatorname{Sym}^{d} E$ that are induced from endomorphisms of $E$. Therefore we need to generalize Theorem 1.3 .

Let $\Phi \in \Gamma\left(\operatorname{End}\left(\operatorname{Sym}^{d} E\right)\right)$ be hermitian with respect to $\operatorname{Sym}^{d} h$. As before, let $g$ be the Fubini-Study metric on $\mathcal{O}_{\mathbb{P} E^{*}}(d)$ induced by the hermitian metric $h$. Define hermitian metrics

$$
h_{t}(\Phi)=\operatorname{Sym}^{d} h(I+t \Phi) \text { and } g_{k}(\Phi)=\widehat{h_{k^{-1}}(\Phi)}
$$

on $\operatorname{Sym}^{d} E$ and $\mathcal{O}_{\mathbb{P} E^{*}}(d)$ respectively. We define the function $F(\Phi) \in$ $C^{\infty}\left(\mathbb{P} E^{*}\right)$ by

$$
F(\Phi)([v])=\left.\left\|v_{d}\right\|_{\mathrm{Sym}^{d} h}^{-2} \frac{d}{d t}\right|_{t=0}\left\|v_{d}\right\|_{h_{t}(\Phi)}^{2}, \quad v \in E^{*} .
$$

Here $v_{d}=v \ldots v$ and note that $\left\|v_{d}\right\|_{\mathrm{Sym}^{d} h}^{2}=\|v\|_{h}^{2 d}$. Simple calculations show that

$$
\begin{gathered}
F(\Phi)([v])=\operatorname{tr}\left(\lambda_{d}\left(v, \operatorname{Sym}^{d} h\right) \Phi\right) . \\
\frac{g_{k}(\Phi)}{g}([v])=1+k^{-1} F(\Phi)+O\left(k^{-2}\right) .
\end{gathered}
$$

Thus,

$$
\omega_{g_{k}(\Phi)}=i \bar{\partial} \partial \log g_{k}(\Phi)=\omega_{g}+k^{-1} i \bar{\partial} \partial F(\Phi)+O\left(k^{-2}\right) .
$$


We define the volume forms $d \mu_{g, \Phi, k}$ on $\mathbb{P} E^{*}$ as follows

$$
d \mu_{g, \Phi, k}=k^{-m} \frac{\left(\omega_{g_{k}(\Phi)}+k \omega\right)^{m+r-1}}{(m+r-1) !} .
$$

For any smooth function $F \in C^{\infty}\left(\mathbb{P} E^{*}\right)$, define

$$
\widetilde{\triangle} F=\frac{(r-1) i \bar{\partial} \partial F \wedge \omega_{g}^{r-2} \wedge \omega^{m}}{\omega_{g}^{r-1} \wedge \omega^{m}} .
$$

Therefore (3.3), (3.15) and (3.16) imply that

$$
g_{k}(\Phi) d \mu_{g, \Phi, k}=\left(1+k^{-1}\left(f_{m-1}+F(\Phi)+\widetilde{\triangle} F(\Phi)\right)+O\left(k^{-2}\right)\right) g d \mu_{g} .
$$

Recall that $d \mu_{g}=\frac{\omega_{g}^{r-1}}{(r-1) !} \wedge \frac{\omega^{n}}{n !}$.

Definition 3.3. Define the bundle map $T: \operatorname{End}\left(\operatorname{Sym}^{d} E\right) \rightarrow \operatorname{End}\left(\operatorname{Sym}^{d} E\right)$ by

$$
\langle s,(T \Phi)(t)\rangle_{\mathrm{Sym}^{d} h}=C_{r, d}^{-1} \int_{\mathbb{P}_{x}^{*}}(F(\Phi)+\widetilde{\triangle} F(\Phi))\langle\widehat{s}, \widehat{t}\rangle_{g} \frac{\omega_{g}^{r-1}}{(r-1) !},
$$

for any $x \in X$ and $s, t \in \operatorname{Sym}^{d} E_{x}$.

We will use the following Lemmas in the proof of Corollary 3.9.

Lemma 3.5. For any $\Phi \in \Gamma\left(\operatorname{End}\left(\operatorname{Sym}^{d} E\right)\right)$ hermitian with respect to Sym $^{d} h$, we have $\operatorname{Tr}(T \Phi)=\operatorname{Tr}(\Phi)$.

Proof. Let $e_{1}, \ldots e_{r}$ be an orthonormal local frame for $E$ with respect to $h$ and $E_{I}$ be the corresponding orthonormal local frame for $\operatorname{Sym}^{d} E$ with respect to $\operatorname{Sym}^{d} h$. We have

$$
\begin{aligned}
\operatorname{Tr}(T \Phi)=\sum_{I}\left\langle E_{I},(T \Phi)\left(E_{I}\right)\right\rangle_{\mathrm{Sym}^{d} h} & =\sum_{I} C_{r, d}^{-1} \int_{\text {Fiber }}(F+\widetilde{\triangle} F)\left\langle\widehat{E_{I}}, \widehat{E_{I}}\right\rangle_{g} \frac{\omega_{g}^{r-1}}{(r-1) !} \\
& =C_{r, d}^{-1} \int_{\text {Fiber }}(F+\widetilde{\triangle} F) \sum_{I}\left|\widehat{E_{I}}\right|_{g}^{2} \frac{\omega_{g}^{r-1}}{(r-1) !} \\
& =C_{r, d}^{-1} \int_{\text {Fiber }}(F+\widetilde{\triangle} F) \frac{\omega_{g}^{r-1}}{(r-1) !} \\
& =C_{r, d}^{-1} \int_{\text {Fiber }} F \frac{\omega_{g}^{r-1}}{(r-1) !} .
\end{aligned}
$$

On the other hand, (3.14) implies that

$$
\int_{\text {Fiber }} F \frac{\omega_{g}^{r-1}}{(r-1) !}=C_{r, d} \operatorname{Tr}(\Phi) \text {. }
$$


Lemma 3.6. For any $\varphi \in \Gamma(\operatorname{End}(E))$, we have $T\left(S^{d} \varphi\right)=S^{d} \varphi$. Conversely, if $T \Phi=\Phi$ for some $\Phi \in \Gamma\left(\operatorname{End}\left(\operatorname{Sym}^{d} E\right)\right)$, then there exists $\varphi \in \Gamma(\operatorname{End}(E))$ such that $\Phi=S^{d} \varphi$. (See (2.2) for definition of Lie algebra homomorphim $S^{d}$.)

Proof. The equations (3.15) and (3.16) imply that

$$
g_{k}(\Phi) \omega_{g_{k}(\Phi)}^{r-1}=\left(1+k^{-1}(F(\Phi)+\widetilde{\triangle} F(\Phi))+O\left(k^{-2}\right)\right) g \omega_{g}^{r-1} .
$$

Therefore

$$
\begin{aligned}
\int_{\mathbb{P} E_{x}^{*}}\langle\widehat{s}, \widehat{t}\rangle_{g_{k}(\Phi) \frac{\omega_{g_{k}(\Phi)}^{r-1}}{(r-1) !}} & =\int_{\mathbb{P} E_{x}^{*}}\left(1+(F(\Phi)+\widetilde{\triangle} F(\Phi)) k^{-1}+O\left(k^{-2}\right)\right)\langle\widehat{s}, \widehat{t}\rangle_{g} \frac{\omega_{g}^{r-1}}{(r-1) !} \\
& =\int_{\mathbb{P} E_{x}^{*}}\langle\widehat{s}, \widehat{t}\rangle_{g} \frac{\omega_{g}^{r-1}}{(r-1) !}+k^{-1} \int_{\mathbb{P} E_{x}^{*}}(F(\Phi)+\widetilde{\triangle} F(\Phi))\langle\widehat{s}, \widehat{t}\rangle_{g} \frac{\omega_{g}^{r-1}}{(r-1) !} \\
& +O\left(k^{-2}\right) .
\end{aligned}
$$

Lemma 2.3 and (3.19) imply that

$C_{r, d}^{-1} \int_{\mathbb{P} E_{x}^{*}}\langle\widehat{s}, \widehat{t}\rangle_{g_{k}(\Phi)} \frac{\omega_{g_{k}(\Phi)}^{r-1}}{(r-1) !}=\langle s, t\rangle_{\mathrm{Sym}^{d} h}+k^{-1}\langle s,(T \Phi)(t)\rangle_{\mathrm{Sym}^{d} h}+O\left(k^{-2}\right)$,

since $g=\widehat{\operatorname{Sym}^{d} h}$. On the other hand, Lemma 2.3 implies that

$$
C_{r, d}^{-1} \int_{\mathbb{P} E_{x}^{*}}\langle\widehat{s}, \widehat{t}\rangle_{g_{k}(\Phi)} \frac{\omega_{g_{k}(\Phi)}^{r-1}}{(r-1) !}=\langle s, t\rangle_{\operatorname{Sym}^{d} h\left(I+k^{-1} \Phi\right)}
$$

if and only if there exists $\varphi \in \Gamma(\operatorname{End}(E))$ such that $\Phi=S^{d} \varphi$. This concludes the proof.

Definition 3.4. Let $h$ be a hermitian metric on $E$ and $\Phi \in \Gamma\left(\operatorname{End}\left(\operatorname{Sym}^{d} E\right)\right)$ be hermitian with respect to $\mathrm{Sym}^{d} h$. We define

$$
\left.\rho_{k}(g, \omega, \Phi):=\rho_{k}\left(g_{k}(\Phi), \omega\right)=\rho_{k}\left(\operatorname{Sym}^{d} \widehat{h\left(I+k^{-1}\right.} \Phi\right), \omega\right),
$$

where $g=\widehat{\operatorname{Sym}^{d} h}$.

In order to prove Theorem 1.4, we need to find an asymptotic expansion for the Bergman kernel $\rho_{k}(g, \omega, \Phi):=\rho_{k}\left(g_{k}(\Phi), \omega\right)$. By definition $\rho_{k}(g, \omega, \Phi)$ is the Bergman kernel of $\mathcal{O}_{\mathbb{P} E^{*}}(d) \otimes L^{k} \rightarrow \mathbb{P} E^{*}$ with respect to the inner product $L^{2}\left(g_{k}(\Phi), d \mu_{g, k, \Phi}\right)$ defined on $H^{0}\left(\mathbb{P} E^{*}, \mathcal{O}_{\mathbb{P} E^{*}}(d) \otimes\right.$ $\left.\pi^{*} L^{k}\right)$. Clearly the $L^{2}-$ inner products $L^{2}\left(g_{k}(\Phi), d \mu_{g, k, \Phi}\right)$ and $L^{2}\left(g_{k}(\Phi) \frac{d \mu_{g, k, \Phi}}{d \mu_{g}}, d \mu_{g}\right)$ are the same. Therefore we can replace the complicated volume form 
$d \mu_{g, k, \Phi}$ by the product volume form $d \mu_{g}$ and the fibre metric $g_{k}(\Phi) \otimes \sigma^{k}$ with $\frac{d \mu_{g, k, \Phi}}{d \mu_{g}} g_{k}(\Phi) \otimes \sigma^{k}$. Then we push forward the metric $g_{k}(\Phi) \frac{d \mu_{g, k, \Phi}}{d \mu_{g}}$ to get a metric $h(k, \Phi)$ on $\operatorname{Sym}^{d} E$ (See Definition 3.5). In order to conclude the theorem, we apply the result on the asymptotics of the Bergman kernel on $\operatorname{Sym}^{d} E$ to the metric $h(k, \Phi)$.

Definition 3.5. We define the hermitian metric $h(k, \Phi)$ on $\operatorname{Sym}^{d} E$ as follows:

$$
h(k, \Phi)=C_{r, d}^{-1} \pi_{*}\left(g_{k}(\Phi) \frac{d \mu_{g, k, \Phi}}{d \mu_{g}} \frac{\omega_{g}^{r-1}}{(r-1) !}\right),
$$

i.e. for any $x \in X$ and $s, t \in \operatorname{Sym}^{d} E_{x}$, we have

$$
\langle s, t\rangle_{h(k, \Phi)}=C_{r, d}^{-1} \int_{\mathbb{P} E_{x}^{*}}\langle\widehat{s}, \widehat{t}\rangle_{g_{k}(\Phi)} \frac{d \mu_{g, k, \Phi}}{d \mu_{g}} \frac{\omega_{g}^{r-1}}{(r-1) !} .
$$

If $h, \omega$ and $\Phi$ vary in a bounded family, then the metrics $h(k, \Phi)$ vary in a bounded family. More precisely, we have the following.

Theorem 3.7. Let $\nu_{0}$ be a fixed Kähler form on $X$ and $h_{0}$ be a fixed hermitian metric on E. For any positive numbers $l$ and $l^{\prime}$ and any positive integer $p$, there exists a positive number $C_{l, l^{\prime}, p}$ such that if

$$
\begin{gathered}
\|\omega\|_{C^{p}\left(\nu_{0}\right)},\|h\|_{C^{p+2}\left(h_{0}, \nu_{0}\right)},\|\Phi\|_{C^{p+2}\left(h_{0}, \nu_{0}\right)} \leq l \text { and } \\
\inf _{x \in X}\|h(x)\|_{h_{0}(x)}, \inf _{x \in X}\left|\omega(x)^{m}\right|_{\nu_{0}(x)} \geq l^{\prime},
\end{gathered}
$$

then $\|h(k, \Phi)\|_{C^{p}\left(h_{0}, \nu_{0}\right)} \leq C_{l, l^{\prime}, p}$, for $k \gg 0$.

We prove Theorem 3.7 in Section 5.

Proof of Theorem 1.4. Recall that

$$
h(k, \Phi)=C_{r, d}^{-1} \pi_{*}\left(g_{k}(\Phi) \frac{d \mu_{g, k, \Phi}}{d \mu_{g}} \frac{\omega_{g}^{r-1}}{(r-1) !}\right) .
$$

Therefore (3.6), (3.7), (3.18) and (3.19) imply that

$$
h(k, \Phi)=\operatorname{Sym}^{d} h\left(I+k^{-1}\left(T(\Phi)+\Psi_{m-1}\right)+O\left(k^{-2}\right)\right) .
$$

Let $B_{k}(h(k, \Phi), \omega)$ be the Bergman kernel of $\operatorname{Sym}^{d} E \otimes L^{k}$ with respect to the $L^{2}$-metric defined by the hermitian metric $h(k, \Phi) \otimes \sigma^{k}$ on $\operatorname{Sym}^{d} E \otimes L^{k}$ and the volume form $\frac{\omega^{m}}{m !}$ on $X$. Therefore, if $s_{1}, \ldots, s_{N}$ is an orthonormal basis for $H^{0}\left(X, \operatorname{Sym}^{d} E \otimes L^{k}\right)$ with respect to the metric $L^{2}\left(h(k) \otimes \sigma^{k}, \frac{\omega^{m}}{m !}\right)$, then

$$
B_{k}(h(k, \Phi), \omega)=\sum s_{i} \otimes s_{i}^{* h(k, \Phi) \otimes \sigma^{k}},
$$


We define $\widetilde{B}_{k}(h, \omega, \Phi)$ as follow

$$
\widetilde{B}_{k}(h, \omega, \Phi)=\sum s_{i} \otimes s_{i}^{* \operatorname{Sym}^{d} h\left(I+k^{-1} \Phi\right) \otimes \sigma^{k}} .
$$

Let $\widehat{s_{1}}, \ldots . \widehat{s_{N}}$ be the corresponding basis for $H^{0}\left(\mathbb{P} E^{*}, \mathcal{O}_{\mathbb{P} E^{*}}(d) \otimes L^{k}\right)$. Hence,

$$
\begin{aligned}
\int_{\mathbb{P} E^{*}}\left\langle\widehat{s_{i}}, \widehat{s_{j}}\right\rangle_{g_{k}(\Phi) \otimes \sigma^{k}} d \mu_{g, \Phi, k} & =\int_{\mathbb{P} E^{*}}\left\langle\widehat{s_{i}}, \widehat{s_{j}}\right\rangle_{g_{k}(\Phi) \otimes \sigma^{k}} \frac{d \mu_{g, \Phi, k}}{d \mu_{g}} d \mu_{g} \\
& =C_{r, d} \int_{X}\left\langle s_{i}, s_{j}\right\rangle_{h(k, \Phi) \otimes \sigma^{k}} \frac{\omega^{m}}{m !} \\
& =C_{r, d} \delta_{i j} .
\end{aligned}
$$

Therefore $\frac{1}{\sqrt{C_{r, d}}} \widehat{s_{1}}, \ldots, \frac{1}{\sqrt{C_{r, d}}} \widehat{s_{N}}$ is an orthonormal basis for $H^{0}\left(\mathbb{P} E^{*}, \mathcal{O}_{\mathbb{P} E^{*}}(d) \otimes\right.$ $\left.L^{k}\right)$ with respect to $L^{2}\left(g_{k}(\Phi) \otimes \sigma^{k}, d \mu_{g, \Phi, k}\right)$. Hence,

$$
C_{r, d} \rho_{k}(g, \Phi, \omega)=C_{r, d} \rho_{k}\left(g_{k}(\Phi), \omega\right)=\sum\left|\hat{s}_{i}\right|_{g_{k}(\Phi)}^{2}
$$

and therefore Lemma 3.4 implies that

$$
\rho_{k}(g, \Phi, \omega)([v])=C_{r, d}^{-1} \operatorname{tr}\left(\lambda_{d}\left(v, \operatorname{Sym}^{d} h\left(I+k^{-1} \Phi\right)\right) \widetilde{B}_{k}(h, \omega, \Phi)\right) .
$$

In order to conclude the proof, it suffices to show that there exist smooth endomorphisms $A_{i}(h, \omega, \Phi) \in \Gamma\left(X, \operatorname{End}\left(\operatorname{Sym}^{d} E\right)\right)$ such that

$$
\widetilde{B}_{k}(h, \omega, \Phi) \sim k^{m}+A_{1}(h, \omega, \Phi) k^{m-1}+\ldots .
$$

The same argument as in the proof of Theorem 1.3 implies that there exist smooth endomorphisms $B_{i} \in \Gamma\left(X, \operatorname{End}\left(\mathrm{Sym}^{d} E\right)\right)$ such that

$$
B_{k}(h(k, \Phi), \omega) \sim k^{m}+B_{1} k^{m-1}+\ldots,
$$

where the first coefficient $B_{i}$ is given by

$$
B_{1}\left(\operatorname{Sym}^{d} h\right)=\frac{i}{2 \pi} \Lambda F_{\left(\operatorname{Sym}^{d} E, \operatorname{Sym}^{d} h\right)}+\frac{1}{2} S(\omega) I_{\operatorname{Sym}^{d} E} \cdot
$$

On the other hand

$$
\begin{aligned}
B_{k}(h(k, \Phi), \omega) & =\sum s_{i} \otimes s_{i}^{{ }^{*} h(k, \Phi) \otimes \sigma^{k}} \\
& =\sum s_{i} \otimes s_{i}^{{ }^{*}{ }^{\operatorname{Sym}^{d} h\left(I+k^{-1} \Phi\right) \otimes \sigma^{k}}}\left(I+k^{-1} \Phi\right)^{-1}\left(I+k^{-1}\left(T \Phi+\Psi_{m-1}\right)+\ldots\right) \\
& =\widetilde{B}_{k}(h, \omega, \Phi)\left(I+k^{-1} \Phi\right)^{-1}\left(I+k^{-1}\left(T \Phi+\Psi_{m-1}\right)+\ldots\right) .
\end{aligned}
$$

Therefore,

$$
\begin{aligned}
\widetilde{B_{k}}(h, \omega, \Phi) & =B_{k}(h(k, \Phi), \omega)\left(I+k^{-1}\left(T \Phi+\Psi_{m-1}\right)+O\left(k^{-2}\right)\right)^{-1}\left(I+k^{-1} \Phi\right) \\
& =B_{k}(h(k, \Phi), \omega)\left(I+k^{-1}\left(\Phi-T \Phi-\Psi_{m-1}\right)+O\left(k^{-2}\right)\right) \\
& \sim k^{m}+\left(B_{1}-\Psi_{m-1}+\Phi-T \Phi\right) k^{m-1}+\ldots
\end{aligned}
$$


Hence (3.12) imply that

$$
\begin{aligned}
A_{1}(h, \omega, \Phi) & =\frac{i r}{2 \pi(r+d)}\left(\Lambda F_{\mathrm{Sym}^{d} h}-\frac{1}{\operatorname{rank}\left(\operatorname{Sym}^{d} E\right)} \operatorname{tr}\left(\Lambda F_{\mathrm{Sym}^{d} h}\right) I\right) \\
& +\frac{1}{2} S(\omega) I+\Phi-T \Phi=A_{1}(h, \omega)+\Phi-T \Phi
\end{aligned}
$$

Notice that Theorem 3.7 implies that if $h, \Phi$ and $\omega$ vary in a bounded family and $\omega, h$ are bounded from below, then the metrics $h(k, \Phi)$ vary in a bounded family. Thus the asymptotic expansion that we obtained for $\widetilde{B}_{k}(h, \omega, \Phi)$ is uniform as long as $h, \Phi$ and $\omega$ vary in a bounded family and $\omega$ and $h$ are bounded from below.

Proposition 3.8. Suppose that $\omega_{\infty} \in 2 \pi c_{1}(L)$ be a Kähler form with constant scalar curvature and $h_{H E}$ be a Hermitian-Einstein metric on $E$, i.e. $\Lambda_{\omega_{\infty}} F_{\left(E, h_{H E}\right)}=\mu I_{E}$, where $\mu$ is the $\omega_{\infty}$-slope of the bundle E. We have

$$
\begin{aligned}
A_{1,1}(\varphi, \eta, \Phi):= & \left.\frac{d}{d t}\right|_{t=0} A_{1}\left(h_{H E}\left(I_{E}+t \varphi\right), \omega_{\infty}+i t \bar{\partial} \partial \eta, I_{S y m^{d} E}+t \Phi\right) \\
= & \mathcal{D}^{*} \mathcal{D} \eta I_{S y m^{d} E}+\frac{i r}{2 \pi(r+d)}\left(S^{d} \Lambda_{\omega_{\infty}} \bar{\partial} \partial \varphi+\Lambda_{\omega_{\infty}}^{2}\left(F_{S y m^{d} h_{H E}} \wedge i \bar{\partial} \partial \eta\right)\right. \\
& \left.\quad-\frac{1}{R} \operatorname{tr}\left(S^{d} \Lambda_{\omega_{\infty}} \bar{\partial} \partial \varphi+\Lambda_{\omega_{\infty}}^{2}\left(F_{h_{H E}} \wedge i \bar{\partial} \partial \eta\right)\right)\right)+\Phi-T \Phi,
\end{aligned}
$$

where $\mathcal{D}^{*} \mathcal{D}$ is Lichnerowicz operator (cf. [D, Page 515]) and $R$ is the rank of the vector bundle Sym ${ }^{d} E$.

Proof. Define $f(t)=\Lambda_{\omega_{\infty}+i t \bar{\partial} \partial \eta} F_{\left(\operatorname{Sym}^{d} h_{\mathrm{HE}}\left(I+t S^{d} \varphi\right)\right)}$. Therefore, we have

$$
m F_{\left(\operatorname{Sym}^{d} h_{\mathrm{HE}}\left(I+t S^{d} \varphi\right)\right)} \wedge\left(\omega_{\infty}+i t \bar{\partial} \partial \eta\right)^{m-1}=f(t)\left(\omega_{\infty}+i t \bar{\partial} \partial \eta\right)^{m} .
$$

Differentiating with respect to $t$ at $t=0$, we obtain

$$
m S^{d} \bar{\partial} \partial \varphi \wedge \omega_{\infty}^{m-1}+m(m-1) F_{\operatorname{Sym}^{d} h_{\mathrm{HE}}} \wedge(i \bar{\partial} \partial \eta) \wedge \omega_{\infty}^{m-2}=f^{\prime}(0) \omega_{\infty}^{m}+m f(0)(i \bar{\partial} \partial \eta) \wedge \omega_{\infty}^{m-1} .
$$

Since $f(0)=\mu I_{E}$, we get

$$
f^{\prime}(0)=S^{d} \Lambda_{\omega_{\infty}} \bar{\partial} \partial \varphi+\Lambda_{\omega_{\infty}}^{2}\left(F_{\mathrm{Sym}^{d} h_{\mathrm{HE}}} \wedge(i \bar{\partial} \partial \eta)\right)-\mu \Lambda_{\omega_{\infty}}(i \bar{\partial} \partial \eta) I_{E} .
$$

On the other hand (cf. [D, pp. 515, 516].)

$$
\left.\frac{d}{d t}\right|_{t=0} S\left(\omega_{\infty}+i t \bar{\partial} \partial \eta\right)=\mathcal{D}^{*} \mathcal{D} \eta
$$


Corollary 3.9. Suppose that $\operatorname{Aut}(X, L) / \mathbb{C}^{*}$ is discrete and $E$ is stable. Then the map

$$
A_{1,1}: \Gamma(\operatorname{End}(E)) \oplus C^{\infty}(X) \oplus \Gamma\left(\operatorname{End}\left(\operatorname{Sym}^{d} E\right)\right) \rightarrow \Gamma_{0}\left(\operatorname{End}\left(\operatorname{Sym}^{d} E\right)\right)
$$

is surjective, where $\Gamma_{0}\left(E n d\left(S y m^{d} E\right)\right)$ is the space of smooth hermitian (with respect to $S_{y m^{d}} h_{H E}$ ) endomorphisms $\Psi$ of $S y m^{d} E$ satisfying $\int_{X} \operatorname{tr}(\Psi) \omega_{\infty}^{m}=0$.

Proof. In this proof we let $F=F_{\left(E, h_{\mathrm{HE}}\right)}$ and $\Lambda=\Lambda_{\omega_{\infty}}$. Define the bundle map $\widetilde{T}: \operatorname{End}\left(\operatorname{Sym}^{d} E\right) \rightarrow \operatorname{End}\left(\operatorname{Sym}^{d} E\right)$ by $\widetilde{T} \Phi=\Phi-T \Phi$. Lemma 3.6 implies that $\operatorname{ker}(\widetilde{T})=S^{d}(\operatorname{End}(E))$. Therefore $\operatorname{ker}(\widetilde{T})$ and $\operatorname{Im}(\widetilde{T})$ are smooth subbundles of $\operatorname{End}\left(\operatorname{Sym}^{d} E\right)$ and as smooth bundles, we have

$$
\operatorname{End}\left(\operatorname{Sym}^{d} E\right)=\operatorname{ker}(\widetilde{T}) \oplus \operatorname{Im}(\widetilde{T}) .
$$

Let $\Psi \in \Gamma_{0}\left(\operatorname{End}\left(\operatorname{Sym}^{d} E\right)\right)$. There exist $\Psi_{1} \in \operatorname{ker}(\widetilde{T})$ and $\Psi_{2} \in \operatorname{Im}(\widetilde{T})$ such that $\Psi=\Psi_{1}+\Psi_{2}$. Hence there exists $\Phi_{0} \in \Gamma\left(\operatorname{End}\left(\operatorname{Sym}^{d} E\right)\right)$ such that

$$
\Psi_{2}=\widetilde{T}\left(\Phi_{0}\right)=\Phi_{0}-T \Phi_{0} .
$$

We know that the map $\eta \in C_{0}^{\infty} \rightarrow \mathcal{D}^{*} \mathcal{D} \eta \in C_{0}^{\infty}$ is surjective since $\operatorname{Aut}(X, L) / \mathbb{C}^{*}$ is discrete (cf. [D, pp. 515, 516]). Thus, we can find $\eta_{0} \in C^{\infty}(X)$ such that $\mathcal{D}^{*} \mathcal{D} \eta_{0}=\frac{1}{R} \operatorname{tr}(\Psi)$. Note that Lemma 3.5 and (3.24) imply that

$$
\mathcal{D}^{*} \mathcal{D} \eta_{0}=\frac{1}{R} \operatorname{tr}(\Psi)=\frac{1}{R} \operatorname{tr}\left(\Psi_{1}\right) .
$$

On the other hand

$\frac{i}{2 \pi}\left(\Lambda^{2}\left(F \wedge i \bar{\partial} \partial \eta_{0}\right)-\frac{1}{R} \operatorname{tr}\left(\Lambda^{2}\left(F \wedge i \bar{\partial} \partial \eta_{0}\right)\right)-\Psi_{1}+\frac{1}{R} \operatorname{tr}\left(\Psi_{1}\right) \in \Gamma_{0}\left(S^{d} \operatorname{End}(E)\right)\right.$.

The map

$$
\varphi \in \Gamma_{0}(\operatorname{End}(E)) \rightarrow \frac{i}{2 \pi} \Lambda \bar{\partial} \partial \varphi \in \Gamma_{0}(\operatorname{End}(E))
$$

is surjective since $E$ is simple (cf. [K]). Therefore, there exists $\varphi_{0} \in$ $\Gamma(\operatorname{End}(E))$ such that

$$
-\frac{i}{2 \pi} S^{d} \Lambda \bar{\partial} \partial \varphi_{0}=\frac{i}{2 \pi}\left(\Lambda^{2}\left(F \wedge i \bar{\partial} \partial \eta_{0}\right)-\frac{1}{R} \operatorname{tr}\left(\Lambda^{2}\left(F \wedge i \bar{\partial} \partial \eta_{0}\right)\right)-\Psi_{1}+\frac{1}{R} \operatorname{tr}\left(\Psi_{1}\right) .\right.
$$

This together with (3.24) imply that

$$
A_{1,1}\left(\varphi_{0}, \eta_{0}, \Phi_{0}\right)=\Psi \text {. }
$$

Note that $\operatorname{tr}\left(\frac{i}{2 \pi} S^{d} \Lambda \bar{\partial} \partial \varphi_{0}\right)=0$. 


\section{Constructing Almost Balanced Metrics}

Let $\sigma_{\infty}$ be a hermitian metric on $L$ such that $\omega_{\infty}=i \bar{\partial} \partial \log \sigma_{\infty}$ is a Kähler form with constant scalar curvature. Let $h_{\mathrm{HE}}$ be the corresponding Hermitian-Einstein metric on $E$, i.e.

$$
\Lambda_{\omega_{\infty}} F_{\left(E, h_{\mathrm{HE}}\right)}=\mu I_{E},
$$

where $\mu$ is the slope of the bundle $E$. Define

$$
\omega_{0}=i \bar{\partial} \partial \log \widehat{S y m}^{d} h_{\mathrm{HE}}=d i \bar{\partial} \partial \log \widehat{h_{\mathrm{HE}}} .
$$

After tensoring by high power of $L$, we can assume without loss of generality that $\omega_{0}$ is a Kähler form on $\mathbb{P} E^{*}$. We fix an integer $a \geq 4$. In order to prove the following, we use ideas introduced by Donaldson in $([\mathrm{D}$, Theorem 26])

Theorem 4.1. Suppose Aut $(X, L)$ is discrete. There exist smooth functions $\eta_{1}, \eta_{2}, \ldots$ on $X$, smooth endomorphisms $\varphi_{1}, \varphi_{2}, \ldots$ of $E$ and $\Phi_{1}, \Phi_{2}, \ldots$ smooth endomorphisms of $S_{y m}^{d} E$ such that for any positive integer $q$ if

$$
\begin{aligned}
& \nu_{k, q}=\omega_{\infty}+i \bar{\partial} \partial\left(\sum_{j=1}^{q} k^{-j} \eta_{j}\right), \\
& h_{k, q}=h_{H E}\left(I_{E}+\sum_{j=1}^{q} k^{-j} \varphi_{j}\right)
\end{aligned}
$$

and

$$
\Phi_{k, q}=I_{S y m^{d} E}+\sum_{j=1}^{q} k^{-j} \Phi_{j},
$$

then

$$
\widetilde{B}_{k}\left(h_{k, q}, \nu_{k, q}, \Phi_{k, q}\right)=\frac{k^{m} C_{r, d} N_{k}}{V_{k}}\left(I_{S y m^{d} E}+\delta_{q}\right),
$$

where $\left\|\delta_{q}\right\|_{C^{a+2}}=O\left(k^{-q-1}\right)$. Here $V_{k}=\operatorname{Vol}\left(\mathbb{P} E^{*}, \mathcal{O}_{\mathbb{P} E^{*}}(d) \otimes L^{k}\right)$ and $N_{k}=h^{0}\left(\mathbb{P} E^{*}, \mathcal{O}_{\mathbb{P} E^{*}}(d) \otimes L^{k}\right)$ are topological invariants.

Proof. The error term in the asymptotic expansion is uniformly bounded in $C^{a+2}$ for all $h, \Phi$ and $\omega$ varying in a bounded family. Therefore there exists a positive integer $s$ depends only on $p$ and $q$ such that

$$
\begin{gathered}
A_{p}(h(1+\varphi), \omega+i \bar{\partial} \partial \eta, I+\Phi)=A_{p}(h, \omega, I)+\sum_{j=1}^{q} A_{p, j}(\varphi, \eta, \Phi) \\
+O\left(\|(\varphi, \eta, \Phi)\|_{C^{s}}^{q+1}\right),
\end{gathered}
$$


where $A_{p, j}$ are homogeneous polynomials of degree $j$, depending on $h$ and $\omega$, in $\varphi, \eta$ and $\Phi$ and its covariant derivatives. Let $\varphi_{1}, \ldots, \varphi_{q}$ be smooth endomorphisms of $E, \Phi_{1}, \ldots, \Phi_{q}$ be smooth endomorphisms of Sym $^{d} E$ and $\eta_{1}, \ldots, \eta_{q}$ be smooth functions on $X$. We have

$$
\begin{gathered}
A_{p}\left(h\left(1+\sum_{j=1}^{q} k^{-j} \varphi_{j}\right), \omega+i \bar{\partial} \partial\left(\sum_{j=1}^{q} k^{-j} \eta_{j}\right), I+\sum_{j=1}^{q} k^{-j} \Phi_{j}\right) \\
=A_{p}(h, \omega, I)+\sum_{j=1}^{q} b_{p, j} k^{-j}+O\left(k^{-q-1}\right)
\end{gathered}
$$

where $b_{p, j}$ 's are multi linear expression on $\varphi_{i}, \Phi_{i}$ 's and $\eta_{i}$ 's. Hence

$$
\begin{aligned}
& \widetilde{B}_{k}\left(h\left(1+\sum_{j=1}^{q} k^{-j} \Phi_{j}\right), \omega+i \bar{\partial} \partial\left(\sum_{j=1}^{q} k^{-j} \eta_{j}\right), I+\sum_{j=1}^{q} k^{-j} \Phi_{j}\right) \\
& =k^{m}+A_{1}(h, \omega, I) k^{m-1}+\ldots . \\
& \quad+\left(A_{q}(h, \omega, I)+b_{q-1,1}+\ldots+b_{1, q-1}\right) k^{m-q}+O\left(k^{m-q-1}\right) .
\end{aligned}
$$

We need to choose $\varphi_{j}, \Phi_{j}$ and $\eta_{j}$ such that coefficients of $k^{m}, \ldots k^{m-q}$ in the right hand side of (4.5) are constant. Donaldson's key observation is that $\eta_{p}, \varphi_{p}$ and $\Phi_{p}$ only appear in the coefficient of $k^{m-p}$ in the form of $A_{1,1}\left(\varphi_{p}, \eta_{p}, \Phi_{p}\right)$. Hence, we can do this inductively. Assume that we choose $\eta_{1}, \eta_{2}, \ldots, \eta_{p-1}, \varphi_{1}, \varphi_{2}, \ldots, \varphi_{p-1}$ and $\Phi_{1}, \Phi_{2}, \ldots, \Phi_{p-1}$ so that the coefficients of $k^{m}, \ldots k^{m-p+1}$ are constant. Now we need to choose $\eta_{p}, \varphi_{p}$ and $\Phi_{p}$ such that the coefficient of $k^{m-p}$ is constant. This means that we need to solve the equation

$$
A_{1,1}\left(\varphi_{p}, \eta_{p}, \Phi_{p}\right)-c_{p} I_{\mathrm{Sym}^{d} E}=P_{p-1},
$$

for $\varphi_{p}, \Phi_{p}, \eta_{p}$ and the constant $c_{p}$. In this equation $P_{p-1}$ is determined by $\varphi_{1}, \ldots, \varphi_{p-1} \Phi_{1}, \ldots, \Phi_{p-1}$ and $\eta_{1}, \ldots, \eta_{p-1}$. Corollary 3.9 implies that we can always solve the equation (4.6).

Corollary 4.2. For any positive integer $q$, there exist hermitian metrics $g_{k, q}$ on $\mathcal{O}_{\mathbb{P} E^{*}}(d)$ and Kähler forms $\nu_{k, q}$ on $X$ in the class of $2 \pi c_{1}(L)$ so that

$$
\rho_{k}\left(g_{k, q}, \nu_{k, q}\right)=\frac{k^{m} N_{k}}{V_{k}}\left(1+\epsilon_{k, q}\right)
$$

where $\left\|\epsilon_{k, q}\right\|_{C^{a+2}}=O\left(k^{-q-1}\right)$. Moreover,

$$
\left\|\omega_{g_{k, q}}+k \nu_{k, q}-\left(\omega_{0}+k \omega_{\infty}\right)\right\|_{C^{a}\left(\omega_{0}+k \omega_{\infty}\right)}=O\left(k^{-1}\right),
$$

where $\omega_{g_{k, q}}=i \bar{\partial} \partial \log g_{k, q}, \omega_{0}$ is defined by (4.1) and $\omega_{\infty}$ is the constant scalar curvature Kähler metric in the class of $2 \pi c_{1}(L)$. 
Proof. Let $H_{k, q}=\operatorname{Sym}^{d} h_{k, q}\left(I+k^{-1} \Phi_{k, q}\right)$ and $g_{k, q}=\widehat{H_{k, q}}$. Lemma 3.4 and Theorem 4.1 imply that

$$
\begin{aligned}
\rho_{k}\left(g_{k, q}, \nu_{k, q}\right) & =\frac{N_{k}}{k^{-m} C_{r, d} V_{k}} \operatorname{Tr}\left(\lambda_{d}\left(v^{*}, H_{k, q}\right)\left(I_{\mathrm{Sym}^{d} E}+\delta_{q}\right)\right) \\
& \left.=\frac{N_{k}}{k^{-m} V_{k}}\left(1+\operatorname{Tr}\left(\lambda_{d}\left(v^{*}, H_{k, q}\right) \delta_{q}\right)\right)\right) .
\end{aligned}
$$

It concludes the first part of corollary, since $H_{k, q}$ is bounded and $\left\|\delta_{k, q}\right\|_{C^{a+2}}=O\left(k^{-q-1}\right)$.

For the second part, define $\widetilde{\omega_{0}}=\omega_{0}+k \omega_{\infty}$ and $g_{k, q}^{\prime}=\widehat{\operatorname{Sym}^{d} h_{k, q}}$. Notice that $g_{k, q}=g_{k, q}^{\prime}\left(\Phi_{k, q}\right)$ (c.f. (3.13)). Now (3.14) implies that

$F\left(\Phi_{k, q}\right)=\operatorname{tr}\left(\lambda_{d} \Phi_{k, q}\right)=\operatorname{tr}\left(\lambda_{d}\left(I+k^{-1} \Phi_{1}+\ldots\right)\right)=1+k^{-1} F\left(\Phi_{1}\right)+O\left(k^{-2}\right)$.

Therefore (3.15) shows that

Hence

$$
\frac{g_{k, q}}{g_{k, q}^{\prime}}=1+k^{-2} F\left(\Phi_{1}\right)+O\left(k^{-3}\right) .
$$

$$
\omega_{g_{k, q}}-\omega_{g_{k, q}^{\prime}}=-k^{-2} i \bar{\partial} \partial F\left(\Phi_{1}\right)+O\left(k^{-3}\right)
$$

which implies that $\left\|\omega_{g_{k, q}}-\omega_{g_{k, q}^{\prime}}\right\|_{C^{a}\left(\omega_{0}\right)}=O\left(k^{-2}\right)$. Thus,

$$
\begin{aligned}
\| \omega_{g_{k, q}}+ & k \nu_{k, q}-\left(\omega_{0}+k \omega_{\infty}\right) \|_{C^{a}\left(\widetilde{\omega_{0}}\right)} \\
& \leq\left\|\omega_{g_{k, q}}-\omega_{g_{k, q}^{\prime}}\right\|_{C^{a}\left(\widetilde{\left.\omega_{0}\right)}\right.}+\left\|\omega_{g_{k, q}}^{\prime}-\omega_{0}\right\|_{C^{a}\left(\widetilde{\omega_{0}}\right)}+k\left\|\nu_{k, q}-\omega_{\infty}\right\|_{C^{a}\left(\widetilde{\omega_{0}}\right)} \\
& \leq\left\|\omega_{g_{k, q}}-\omega_{g_{k, q}^{\prime}}\right\|_{C^{a}\left(\omega_{0}\right)}+\left\|\omega_{g_{k, q}}^{\prime}-\omega_{0}\right\|_{C^{a}\left(\omega_{0}\right)}+k\left\|\nu_{k, q}-\omega_{\infty}\right\|_{C^{a}\left(k \omega_{\infty}\right)} \\
& =\left\|\omega_{g_{k, q}}-\omega_{g_{k, q}^{\prime}}\right\|\left\|_{C^{a}\left(\omega_{0}\right)}+\right\| \omega_{g_{k, q}}^{\prime}-\omega_{0}\left\|_{C^{a}\left(\omega_{0}\right)}+\right\| \nu_{k, q}-\omega_{\infty} \|_{C^{a}\left(\omega_{\infty}\right)} \\
& =O\left(k^{-1}\right) .
\end{aligned}
$$

Notice that by definition, we have

$$
\begin{aligned}
& \left\|\omega_{g_{k, q}}^{\prime}-\omega_{0}\right\|_{C^{a}\left(\omega_{0}\right)}=O\left(k^{-1}\right), \\
& \left\|\nu_{k, q}-\omega_{\infty}\right\|_{C^{a}\left(\omega_{\infty}\right)}=O\left(k^{-1}\right) .
\end{aligned}
$$

\section{Proof of Theorem 3.1] AND 3.7}

The goal of this section is to prove theorem 3.1 and Theorem 3.7. In this section, we fix a background metric $h_{0}$ on $E$ and a Kähler form $\nu_{0}$ on $X$. We denote the chern connections on $E$ and $\operatorname{Sym}^{d} E$ with respect to $h_{0}$ and $\operatorname{Sym}^{d} h_{0}$ by $\nabla$. All norms are with respect to $h_{0}, \mathrm{Sym}^{d} h_{0}$ and $\nu_{0}$. In this section we use the multi-index notation as follows:

For a multi-index $I=\left(i_{1}, \ldots, i_{r}\right)$, define $|I|=i_{1}+\cdots+i_{r}$ and $\lambda^{I}=\lambda_{1}^{i_{1}} \ldots \lambda_{r}^{i_{r}}$. Let $h$ be a hermitian metric on $E$ and $e_{1}, \ldots, e_{r}$ be a 
local holomorphic frame for $E$. We define $h_{i j}=\left\langle e_{i}, e_{j}\right\rangle_{h}$ and denote the inverse of the matrix $\left(h_{i j}\right)$ by $\left(h^{i j}\right)$. The hermitian metric $h$ on $E$ induces a hermitian metric $\mathrm{Sym}^{d} h$ on $\mathrm{Sym}^{d} E$. Any multi-index $I=\left(i_{1}, \ldots, i_{r}\right)$ such that $|I|=d$ defines a local holomorphic section $e^{I}=e_{1}^{i_{1}} \ldots e_{r}^{i_{r}}$ and the set $\left\{e^{I}|| I \mid=d\right\}$ is a holomorphic local frame for $\operatorname{Sym}^{d} E$. Define $h_{I J}=\left\langle e^{I}, e^{J}\right\rangle_{\operatorname{Sym}^{d} h}$ and as before the matrix $\left(h^{I J}\right)$ is the inverse of the matrix $\left(h_{I J}\right)$.

Definition 5.1. A smooth function $f$ on $\mathbb{P} E^{*}$ is called homogenous of order $k$ with respect to the hermitian metric $h$ if there exists a local holomorphic frame $\underline{e}=\left(e_{1}, \ldots, e_{r}\right)$ on $E$ and smooth functions $f_{I J}$ on $X$ such that

$$
f(\lambda)=\frac{\sum_{|I|=|J|=k} f_{I J} \lambda^{I} \bar{\lambda}^{J}}{\left(\sum h^{i j} \lambda_{i} \overline{\lambda_{j}}\right)^{k}},
$$

where $\lambda_{1}, \ldots \lambda_{r}$ are the homogenous coordinates on the fibres with respect to the local frame $\underline{e}$. We define $\|f\|_{p, \underline{e}, h}=\max _{I, J}\left\|F_{I J}\right\|_{c^{p}}$

From now on, let $h$ be a hermitian metric on $E$ and $g=\widehat{\operatorname{Sym}^{d} h}$ be the induced metric on $\mathcal{O}_{\mathbb{P} E^{*}}(d)$. The smooth functions $f_{1}, \ldots f_{m}$ on $\mathbb{P} E^{*}$ and smooth hermitian endomorphisms $\Psi_{1}, \ldots \Psi_{m}$ of $\operatorname{Sym}^{d} E$ are defined in section 3 (See (3.3) and (3.7)) The first step to prove Theorem 3.1 is to estimate $\left\|f_{1}\right\|_{p, \underline{e}, h}, \ldots\left\|f_{m}\right\|_{p, \underline{e}, h}$. We establish such an estimate in Proposition 5.3. The second step is to find an upper bound for $\left\|\Psi_{i}\right\|_{p, \underline{e}}$ in terms of $\left\|f_{i}\right\|_{p, \underline{e}}$. This is the content of Theorem 5.4.

The following lemma is straightforward since $X$ is compact.

Lemma 5.1. For any $x \in X$, there exists a holomorphic local frame $e_{1}, \ldots, e_{r}$ on $E$ around $x$ such that

$$
\frac{1}{2} \leq\left\|\left[\left\langle e_{i}, e_{j}\right\rangle_{h_{0}}\right]\right\|_{o p} \leq 2 \quad \text { and } \quad\left\|\nabla^{k} e_{i}\right\| \leq C(p) \quad k=0, \ldots, p,
$$

where $C(p)$ is a constant depends only on $p, \nu_{0}$ and $h_{0}$.

Lemma 5.2. ([S, Lem. 5.2$]$ ) For any positive integer $p$ there exists a constant $C$ such that for any $(j, j)$-form $\gamma$, we have

$$
\left\|\nabla^{p}\left(\Lambda^{j} \gamma\right)\right\|_{\nu_{0}} \leq \frac{C}{\inf _{x \in X}\left|\omega(x)^{m}\right|_{\nu_{0}(x)}}\left(\|\gamma\|_{C^{p}\left(\nu_{0}\right)}+\left\|\Lambda^{j} \gamma\right\|_{C^{p-1}\left(\nu_{0}\right)}\right)\left(\sum_{i=1}^{m}\|\omega\|_{C^{p}\left(\nu_{0}\right)}^{i}\right)
$$

where $\nabla$ is the connection defined with respect to $\nu_{0}$.

Proposition 5.3. For any $1 \leq j \leq m$, the function $f_{j}$ is homogenous of order $j$ with respect to $h$ (For definition of $f_{j}$, see (3.3)). 
Moreover there exists a constant $C$ depends only on $p$ and $m$ such that for any local holomorphic frame $\underline{e}=\left(e_{1}, \ldots e_{r}\right)$ satisfying (5.1), we have

$$
\left\|f_{i}\right\|_{p, \underline{e, h}} \leq C \max \left(\left\|\frac{\nu_{0}^{m}}{\omega^{m}}\right\|_{C^{0}}^{p}, 1\right)\|h\|_{C^{p+2}}^{j}\left(\sum_{i=0}^{m}\|\omega\|_{C^{p}}^{i}\right)^{p+1}
$$

Proof. Let $e_{1}, \ldots, e_{r}$ be a local holomorphic frame for $E$ around $p$. Define $h_{i j}=\left\langle e_{i}, e_{j}\right\rangle_{h}$ and $\frac{i}{2 \pi} F_{h}=\left(\omega_{i j}\right)$. Let $\lambda_{1}, \ldots, \lambda_{r}$ be the homogeneous coordinates on the fibre. We have

$$
\omega_{g}=\omega_{\mathrm{FS}, g}+\frac{\sum \omega_{i j} \lambda_{i} \overline{\lambda_{j}}}{\sum h^{i j} \lambda_{i} \bar{\lambda}_{j}}
$$

Therefore, $\omega_{g}^{r+j-1} \wedge \omega^{m-j}=\left(\begin{array}{c}r+j-1 \\ r-1\end{array}\right) \omega_{\mathrm{FS}, g}^{r-1} \wedge\left(\frac{\sum \omega_{i j} \lambda_{i} \overline{\lambda_{j}}}{\sum h^{i j} \lambda_{i} \overline{\lambda_{j}}}\right)^{j} \wedge \omega^{m-j}$.

The definition of $f_{m-j}$ gives

$$
f_{m-j} \omega_{g}^{r-1} \wedge \omega^{m}=\left(\begin{array}{c}
m \\
j
\end{array}\right) \omega_{g}^{r-1} \wedge\left(\left(\frac{\sum \omega_{i j} \lambda_{i} \overline{\lambda_{j}}}{\sum h^{i j} \lambda_{i} \overline{\lambda_{j}}}\right)^{j} \wedge \omega^{m-j}\right)
$$

Hence

$$
f_{m-j} \omega_{\mathrm{FS}, g}^{r-1} \wedge \omega^{m}=\left(\begin{array}{c}
m \\
j
\end{array}\right) \omega_{\mathrm{FS}, g}^{r-1} \wedge\left(\left(\frac{\sum \omega_{i j} \lambda_{i} \overline{\lambda_{j}}}{\sum h^{i j} \lambda_{i} \bar{\lambda}_{j}}\right)^{j} \wedge \omega^{m-j}\right) .
$$

Therefore,

$$
\omega_{\mathrm{FS}, g}^{r-1} \wedge\left(f_{m-j} \omega^{m}-\left(\begin{array}{c}
m \\
j
\end{array}\right)\left(\frac{\sum \omega_{i j} \lambda_{i} \overline{\lambda_{j}}}{\sum h^{i j} \lambda_{i} \overline{\lambda_{j}}}\right)^{j} \wedge \omega^{m-j}\right)=0,
$$

which implies

$$
f_{m-j} \omega^{m}=\left(\begin{array}{c}
m \\
j
\end{array}\right)\left(\frac{\sum \omega_{i j} \lambda_{i} \overline{\lambda_{j}}}{\sum h^{i j} \lambda_{i} \overline{\lambda_{j}}}\right)^{j} \wedge \omega^{m-j}=\left(\begin{array}{c}
m \\
j
\end{array}\right) \frac{\sum_{I, J} \Omega_{I J} \lambda^{I} \bar{\lambda}^{J}}{\left(\sum h^{i j} \lambda_{i} \bar{\lambda}_{j}\right)^{j}} \wedge \omega^{m-j} .
$$

Thus,

$$
f_{m-j}=\frac{\sum_{I, J} \Lambda^{j} \Omega_{I J} \lambda^{I} \bar{\lambda}^{J}}{\left(\sum h^{i j} \lambda_{i} \bar{\lambda}_{j}\right)^{j}} .
$$

There exists a constant $C$ depends only on $p$ and $m$ such that

$$
\left\|\Omega_{I J}\right\|_{C^{p}} \leq C \max _{i, j}\left\|\omega_{i j}\right\|_{C^{p}}^{j} \leq C\|h\|_{C^{p+2}}^{j} .
$$

Applying Lemma 5.2, we obtain

$$
\left\|\Lambda^{j} \Omega_{I J}\right\|_{C^{p}} \leq C \max \left(\left\|\frac{\nu_{0}^{m}}{\omega^{m}}\right\|_{C^{0}}, 1\right)\left(\|h\|_{C^{p+2}}^{j}+\left\|\Lambda^{j} \Omega_{I J}\right\|_{C^{p-1}}\right)\left(\sum_{i=0}^{m}\|\omega\|_{C^{p}}^{i}\right) .
$$


Note that

$$
\left(\inf _{x \in X}\left|\frac{\omega(x)^{m}}{\nu_{0}(x)^{m}}\right|\right)^{-1}=\sup _{x \in X}\left|\frac{\nu_{0}(x)^{m}}{\omega(x)^{m}}\right|=|| \frac{\nu_{0}^{m}}{\omega^{m}}||_{C^{0}} .
$$

Hence induction on $p$ concludes the proof.

Definition 5.2. For any smooth function $f$ on $\mathbb{P} E^{*}$, there exists a unique endomorphism $\Psi(f) \in E n d\left(\operatorname{Sym}^{d} E\right)$ which is hermitian with respect to $\mathrm{Sym}^{d} h$ defined as follows:

$$
\langle s, \Psi(f) t\rangle_{\operatorname{Sym}^{d} h}=C_{r, d}^{-1} \int_{\mathbb{P} E_{x}^{*}} f\langle\hat{s}, \hat{t}\rangle_{g} \frac{\omega_{g}^{r-1}}{(r-1) !},
$$

for any $x \in M$ and $s, t \in \operatorname{Sym}^{d} E_{x}$. Here $g=\widehat{\operatorname{Sym}^{d} h}$ is the induced metric on $\mathcal{O}_{\mathbb{P} E^{*}}(d)$ by $h$. Note that we have $\Psi_{i}=\Psi\left(f_{i}\right)$.

Definition 5.3. Let $\Psi$ be an endomorphism of $\operatorname{Sym}^{d} E$ and $\underline{e}=\left(e_{1}, \ldots, e_{r}\right)$ be a local holomorphic coordinate on $E$. Then there exist local functions $\Psi_{I J}$ on $X$ such that

$$
\Psi e^{I}=\sum_{J} \Psi_{I J} e^{J}
$$

We define

$$
\|\Psi\|_{p, \underline{e}}=\max \left\|\Psi_{I J}\right\|_{c^{p}} .
$$

Theorem 5.4. There exists a constant $C$ depends only on $p$ such that for any local holomorphic frame $\underline{e}=\left(e_{1}, \ldots, e_{r}\right)$ satisfying (5.1) and any smooth homogeneous function $f$ of order $k$ on $\mathbb{P} E^{*}$, we have

$$
\|\Psi(f)\|_{C^{p}} \leq C\|f\|_{p, \underline{e}}
$$

The proof follows from the following lemmas.

Lemma 5.5. There exists a constant $C$ depends only on $p$ such that for any local holomorphic frame $\underline{e}=\left(e_{1}, \ldots, e_{r}\right)$ and any smooth homogeneous function $f$ of order $N$ on $\mathbb{P} E^{*}$, we have

$$
\|\Psi(f)\|_{p, \underline{e}} \leq C\|f\|_{p, \underline{e}} .
$$

Proof. We have

$$
\Psi(f) e^{I}=\sum_{J} \Psi_{I J} e^{J} .
$$

Let $\lambda_{1}, \ldots, \lambda_{r}$ be the homogeneous coordinates on the fibre. Suppose that

$$
f(\lambda)=\frac{\sum f_{I J} \lambda^{I} \bar{\lambda}^{J}}{\left(\sum h^{i j} \lambda_{i} \overline{\lambda_{j}}\right)^{N}} .
$$


Therefore

$$
\begin{aligned}
\Psi_{I J} & =\int f(\lambda) \frac{\lambda^{I} \bar{\lambda}^{J}}{\left(\sum h^{i j} \lambda_{i} \overline{\lambda_{j}}\right)^{d}} \frac{\omega_{g}^{r-1}}{(r-1) !} \\
& =\sum f_{K L} \int \frac{\lambda^{K} \bar{\lambda}^{L} \lambda^{I} \bar{\lambda}^{J}}{\left(\sum h^{i j} \lambda_{i} \overline{\lambda_{j}}\right)^{d+N}} \frac{\omega_{g}^{r-1}}{(r-1) !} \\
& =\sum f_{K L} c_{K L I J},
\end{aligned}
$$

Here

$$
c_{K L I J}=\int \frac{\lambda^{K} \bar{\lambda}^{L} \lambda^{I} \bar{\lambda}^{J}}{\left(\sum h^{i j} \lambda_{i} \overline{\lambda_{j}}\right)^{d+N}} \frac{\omega_{g}^{r-1}}{(r-1) !} .
$$

An easy computation shows that $\left|C_{K L I J}\right| \leq 1$. Thus

$$
\left\|\Psi_{I J}\right\|_{c^{p}} \leq C \max \left\|f_{K L}\right\|_{c^{p}}=C\|f\|_{p, \underline{e}}
$$

Lemma 5.6. There exists a constant $C$ depends only on $p$ such that for any local holomorphic frame $\underline{e}=\left(e_{1}, \ldots, e_{r}\right)$ satisfying (5.1) and smooth endomorphism $\Psi$ of $S_{y m}^{d} E$, we have

$$
\|\Psi\|_{C^{p}} \leq C\|\Psi\|_{p, \underline{e}} .
$$

Proof. We have

$$
\Psi e^{I}=\sum_{J} \Psi_{I J} e^{J}
$$

Applying $\nabla^{p}$, we get

$$
\sum_{i=0}^{p} \nabla^{i} \Psi \nabla^{p-i} e^{I}=\sum_{J} \sum_{i=0}^{p} \nabla^{i} \Psi_{I J} \nabla^{p-i} e^{J}
$$

This implies that

$$
\left(\nabla^{p} \Psi\right) e^{I}=-\sum_{i=0}^{p-1} \nabla^{i} \Psi \nabla^{p-i} e^{I}+\sum_{J} \sum_{i=0}^{p} \nabla^{i} \Psi_{I J} \nabla^{p-i} e^{J}
$$

Therefore, 


$$
\begin{aligned}
\|\left(\nabla^{p} \Psi\right) e^{I} \mid & \leq \sum_{i=0}^{p-1}\left\|\nabla^{i} \Psi\right\|\left\|\nabla^{p-i} e^{I}\right\|+\sum_{J} \sum_{i=0}^{p}\left\|\nabla^{i} \Psi_{I J}\right\|\left\|\nabla^{p-i} e^{J}\right\| \\
& \leq C(p)\left(\sum_{i=0}^{p-1}\left\|\nabla^{i} \Psi\right\|+\sum_{J} \sum_{i=0}^{p}\left\|\Psi_{I J}\right\|_{C^{p}}\right) \\
& \leq C C(p)\left(\sum_{i=0}^{p-1}\left\|\nabla^{i} \Psi\right\|+\mid \Psi \|_{p, \underline{e}}\right) .
\end{aligned}
$$

Thus

$$
\|\Psi\|_{C^{p}} \leq C\left(\|\Psi\|_{C^{p-1}}+\|\Psi\|_{p, \underline{e}}\right) .
$$

Now we can conclude the lemma by induction on $p$.

Proof of Thm. 3.1. applying Proposition 5.3 and Theorem 5.4, we have

$$
\begin{aligned}
\left\|\Psi_{i}\right\|_{C^{p}} & =\left\|\Psi\left(f_{i}\right)\right\|_{C^{p}} \leq C\left\|f_{i}\right\|_{p, \underline{e}, h} \\
& \leq C \max \left(\left\|\frac{\nu_{0}^{m}}{\omega^{m}}\right\|_{C^{0}}^{p}, 1\right)\|h\|_{C^{p+2}}^{j}\left(\sum_{i=0}^{m}\|\omega\|_{C^{p}}^{i}\right)^{p+1} .
\end{aligned}
$$

This concludes the proof.

Let $\Phi \in \Gamma\left(\operatorname{End}\left(\operatorname{Sym}^{d} E\right)\right)$ be hermitian with respect to $\operatorname{Sym}^{d} h$. As in section 3, we can define hermitian metrics $h_{t}(\Phi)=\operatorname{Sym}^{d} h(I+t \Phi)$ and $g_{k}(\Phi)=\widehat{h_{k^{-1}}(\Phi)}$ on $\operatorname{Sym}^{d} E$ and $\mathcal{O}_{\mathbb{P} E^{*}}(d)$. In the rest of this section $C$ denotes a constant depends only on $p, d, m, r, \nu_{0}$ and $h_{0}$ that might change from line to line.

Lemma 5.7. There exists a constant $C$ depends only on $p, d, m, r, \nu_{0}$ and $h_{0}$ such that

$$
\left\|\frac{g_{k}(\Phi)}{g}-1\right\|_{C^{p}} \leq C\|\Phi\|_{C^{p}}\|h\|_{C^{p}}^{d}\left(\inf _{x \in X}\|h(x)\|_{h_{0}(x)}\right)^{-2 d}
$$

for $k \gg\|\Phi\|_{C^{p}}$.

Proof. A straightforward computation Shows that

$$
\frac{g}{g_{k}(\Phi)}=\sum_{i=0}^{\infty}(-1)^{i} k^{-i} \operatorname{tr}\left(\lambda_{d} \Phi^{i}\right) .
$$


Let $e_{1}, \ldots e_{r}$ be a holomorphic local frame for $E$ satisfying (5.1). Suppose that $\Phi e^{I}=\sum_{J} \phi_{I J} e^{J}$, then

$$
\operatorname{tr}\left(\lambda_{d} \Phi^{\alpha}\right)=\frac{\sum \lambda^{I_{0}} \overline{\lambda^{K}} h^{I_{1} K} \phi_{I_{1} I_{2}} \ldots \phi_{I_{\alpha} I_{0}}}{\left(\sum_{i j} h^{i j} \lambda_{i} \overline{\lambda_{j}}\right)^{d}} .
$$

Therefore

$$
\begin{aligned}
\left\|\operatorname{tr}\left(\lambda_{d} \Phi^{\alpha}\right)\right\| \|_{C^{p}} & \leq C^{\alpha}\|\Phi\|_{C^{p}}^{\alpha} \sup _{I J}\left\|h_{I J}\right\|_{C^{p}} \sup _{I J}\left\|h^{I J}\right\|_{C^{0}}^{2} \\
& \leq C^{\alpha}\|\Phi\|_{C^{p}}^{\alpha}\|h\|_{C^{p}}^{d}\left(\inf _{x \in X}\|h(x)\|_{h_{0}(x)}\right)^{-2 d} .
\end{aligned}
$$

Therefore

$$
\begin{aligned}
\left\|\frac{g_{k}(\Phi)}{g}-1\right\|_{C^{p}} & \leq \sum_{i=1}^{\infty} k^{-i}\left\|\operatorname{tr}\left(\lambda_{d} \Phi^{i}\right)\right\|_{C^{p}} \\
& \leq\|h\|_{C^{p}}^{d}\left(\inf _{x \in X}\|h(x)\|_{h_{0}(x)}\right)^{-2 d} \sum_{i=1}^{\infty} C^{i} k^{-i}\|\Phi\|_{C^{p}}^{i} \\
& =\|h\|_{C^{p}}^{d}\left(\inf _{x \in X}\|h(x)\|_{h_{0}(x)}\right)^{-2 d} \frac{C k^{-1}\|\Phi\|_{C^{p}}}{1-C k^{-1}\|\Phi\|_{C^{p}}} .
\end{aligned}
$$

This concludes the proof since

$$
\left\|\frac{g}{g_{k}(\Phi)}\right\|_{C^{p}} \sim 1 \text { for } k \gg\|\Phi\|_{C^{p}}
$$

Lemma 5.8. Let $H$ be a hermitian metric on $S_{y m}^{d} E$ and $g=\widehat{H}$ be the induced metric on $\mathcal{O}_{\mathbb{P} E^{*}}(d)$. Let $\chi_{1}$ and $\chi_{2}$ be smooth functions on $\mathbb{P} E^{*}$. Define hermitian metrics $H_{1}$ and $H_{2}$ on $S^{d}{ }^{d} E$ as follows:

$$
\langle s, t\rangle_{H_{i}}=\int_{\mathbb{P} E_{x}^{*}} \chi_{i}\langle\hat{s}, \hat{t}\rangle_{g} \omega_{g}^{r-1},
$$

for any $x \in M$ and $s, t \in \operatorname{Sym}^{d} E_{x}$. There exists a constant $C$ depends only on $p, d, m, r, \nu_{0}$ and $h_{0}$ such that

$$
\left\|H_{1}-H_{2}\right\|_{C^{p}} \leq C\left\|\chi_{1}-\chi_{2}\right\|_{C^{p}}\|h\|_{C^{p}}^{r}\left(\inf _{x \in X}\|h(x)\|_{h_{0}(x)}\right)^{-2} .
$$

Proof. Let $e_{1}, \ldots e_{r}$ be a holomorphic local frame for $E$ satisfying (5.1). Then

$$
\left\langle\widehat{e^{I}}, \widehat{e^{J}}\right\rangle_{g} \omega_{g}^{r-1}=d \frac{\operatorname{det}\left(h_{i j}\right) \sum \lambda^{I} \overline{\lambda^{J}}}{\left(\sum_{i j} h^{i j} \lambda_{i} \overline{\lambda_{j}}\right)^{r}} d \lambda \wedge d \bar{\lambda} .
$$

Therefore 


$$
\begin{aligned}
\left\|H_{2}-H_{1}\right\|_{C^{p}} & \leq C \sum\left\|\int_{\text {Fibre }} \chi_{1}\left\langle\widehat{e^{I}}, \widehat{e^{J}}\right\rangle_{g} \omega_{g}^{r-1}-\int_{\text {Fibre }} \chi_{2}\left\langle\hat{E}_{i}, \hat{E}_{j}\right\rangle_{g} \omega_{g}^{r-1}\right\|_{C^{p}} \\
& \leq C\left\|\chi_{1}-\chi_{2}\right\|_{C^{p}}\left\|\operatorname{det}\left(h_{i j}\right)\right\| C_{C^{p}}\left\|h^{i j}\right\|_{C^{0}} \\
& \leq C\left\|\chi_{1}-\chi_{2}\right\|_{C^{p}}\|h\|_{C^{p}}^{r}\left(\inf _{x \in X}\|h(x)\|_{h_{0}(x)}\right)^{-2} .
\end{aligned}
$$

Proof of Theorem 3.7. In this proof $C_{l, l^{\prime}}$ denotes a constant depends only on $p, d, m, r, l, l^{\prime} \nu_{0}$ and $h_{0}$ that might change from line to line. Lemma 5.7 implies that

$$
\left\|\frac{g_{k}(\Phi)}{g}-1\right\|_{C^{p+2}} \leq\|\Phi\|_{C^{p+2}}\|h\|_{C^{p+2}}^{d}\left(\inf _{x \in X}\|h(x)\|_{h_{0}(x)}\right)^{-2 d} \leq C l^{d+1} l^{2} k^{-1}
$$

as long as $k \gg l$. Hence

$$
\begin{aligned}
\left\|\left(\omega_{g_{k}(\Phi)}+k \omega\right)-\left(\omega_{g}+k \omega\right)\right\|_{C^{p}} & =\left\|i \bar{\partial} \partial \log \frac{g_{k}(\Phi)}{g}\right\|_{C^{p}} \leq\left\|\log \frac{g_{k}(\Phi)}{g}\right\|_{C^{p+2}} \\
& \leq-\log \left(1-C\left\|\frac{g_{k}(\Phi)}{g}-1\right\|_{C^{p+2}}\right) \\
& \leq C\left\|\frac{g_{k}(\Phi)}{g}-1\right\|_{C^{p+2}} \leq C_{l, l^{\prime}} k^{-1} .
\end{aligned}
$$

This implies that

$$
\left\|\frac{d \mu_{g, k, \Phi}-d \mu_{g, k}}{d \mu_{g, k}}\right\|_{C^{p}} \leq C_{l, l^{\prime}} k^{-1} .
$$

Hence

$$
\begin{aligned}
\left\|\frac{g_{k}(\Phi)}{g} \frac{d \mu_{g, k, \Phi}}{d \mu_{g}}-\frac{d \mu_{g, k, \Phi}}{d \mu_{g}}\right\|_{C^{p}} & \leq C\left\|\frac{g_{k}(\Phi)}{g}-1\right\|\left\|_{C^{p}}\right\| \frac{d \mu_{g, k, \Phi}}{d \mu_{g, k}} \|_{C^{p}} \\
& +\left.\left\|\frac{d \mu_{g, k, \Phi}}{d \mu_{g, k}}-\frac{d \mu_{g, k, \Phi}}{d \mu_{g, k}}\right\|\right|_{C^{p}}\left\|\frac{g_{k}(\Phi)}{g}\right\|_{C^{p}}\left\|\frac{d \mu_{g, k}}{d \mu_{g}}\right\|_{C^{p}} \\
& \leq C_{l, l^{\prime}} k^{-1} .
\end{aligned}
$$

Note that

$$
\left\|\frac{d \mu_{g, k}}{d \mu_{g}}\right\|_{C^{p}} \sim 1 \text { for } k \gg 0 .
$$

Now, applying Lemma 5.8 to the functions $\chi_{1}=\frac{d \mu_{g, k}}{d \mu_{g}}$ and $\chi_{2}=$ $\frac{d \mu_{g, k, \Phi}}{d \mu_{g, k}} \frac{g_{k}(\Phi)}{g}$, we get

$$
\|h(k, \Phi)-h(k)\|_{C^{p}} \leq C_{l, l^{\prime}} \mid \chi_{1}-\chi_{2} \|_{C^{p}} \leq C_{l, l^{\prime}} k^{-1} .
$$

This concludes the proof. 


\section{Proof of the MAIN THEOREM}

In this section, we prove Thm. 1.2, In order to do that, we want to apply [S, Theorem 4.6.]. Hence, we need to construct a sequence of almost balanced metrics on $\mathbb{P} E^{*}, \mathcal{O}_{\mathbb{P} E^{*}}(d) \otimes L^{\otimes k}$. In order to apply [S, Theorem 4.6.], we also need the following.

Proposition 6.1. ([S, Prop. 7.1$]$ ) Let $E$ be a holomorphic vector bundle over a compact Kähler manifold $X$. Suppose that $X$ has no nonzero holomorphic vector fields. If $E$ is stable, then $\mathbb{P} E^{*}$ has no nontrivial holomorphic vector fields.

Proof of Thm. 1.2. Since Chow stability is equivalent to the existence of balanced metric, it suffices to show that $\left(\mathbb{P} E^{*}, \mathcal{O}_{\mathbb{P} E^{*}}(d) \otimes \pi^{*} L^{k}\right)$ admits balanced metric for $k \gg 0$. Fix a positive integer $q$. From now on we drop all indexes $q$ for simplicity. Let $\omega_{\infty}$ be a constant scalar curvature Kähler metric on $X$ and $\sigma_{\infty}$ be a hermitian metric on $L$ such that $i \bar{\partial} \partial \log \sigma_{\infty}=\omega_{\infty}$. Define $\sigma_{k}=\sigma_{\infty} e^{\sum_{j=1}^{q} k^{-j} \eta_{j}}$, where $\eta_{j}$ 's are given by Thm. 4.1. Therefore $i \bar{\partial} \partial \log \sigma_{k}=\nu_{k, q}$. For the rest of the proof, we denote $\nu_{k, q}$ by $\nu_{k}$. Let $t_{1}, \ldots, t_{N}$ be an orthonormal basis for $H^{0}\left(\mathbb{P} E^{*}, \mathcal{O}_{\mathbb{P} E^{*}}(d) \otimes L^{k}\right)$ with respect to $L^{2}\left(g_{k} \otimes \sigma_{k}^{\otimes k}, \frac{\left(\omega_{g_{k}}+k \nu_{k}\right)^{m+r-1}}{(m+r-1) !}\right)$. Thus, Cor. 4.2 implies

$$
\sum\left|t_{i}\right|_{g_{k} \otimes \sigma_{k}^{\otimes k}}^{2}=\frac{N_{k}}{V_{k}}\left(1+\epsilon_{k}\right) .
$$

Define $g_{k}^{\prime}=\frac{V_{k}}{N_{k}}\left(1+\epsilon_{k}\right)^{-1} g_{k}$. We have $\sum\left|t_{i}\right|_{g_{k}^{\prime} \otimes \sigma_{k}^{\otimes k}}^{2}=1$. This implies that the metric $g_{k}^{\prime}$ is the Fubini-Study metric on $\mathcal{O}_{\mathbb{P} E^{*}}(d) \otimes L^{k}$ induced by the embedding $\iota_{\underline{t}}: \mathbb{P} E^{*} \rightarrow \mathbb{P}^{N-1}$, where $\underline{t}=\left(t_{1}, \ldots, t_{N}\right)$. We prove that this sequence of embedding is almost balanced of order $q$, i.e

$$
\int_{\mathbb{P} E^{*}}\left\langle t_{i}, t_{j}\right\rangle_{g_{k}^{\prime} \otimes \sigma_{k}^{\otimes k}} \frac{\left(\omega_{g_{k}^{\prime}}+k \nu_{k}\right)^{m+r-1}}{(m+r-1) !}=D^{(k)} \delta_{i j}+M_{i j},
$$

where $M^{(k)}=\left[M_{i j}\right]$ is a trace free hermitian matrix, $D^{(k)}=\frac{V_{k}}{N_{k}} \rightarrow C_{r, d}$ as $k \rightarrow \infty$ and $\left\|M^{(k)}\right\|_{\text {op }}=O\left(k^{-q-1}\right)$. We have

$$
\begin{aligned}
M_{i j}^{(k)} & =\int_{\mathbb{P} E^{*}}\left\langle t_{i}, t_{j}\right\rangle_{g_{k}^{\prime} \otimes \sigma_{k}^{\otimes k}} \frac{\left(\omega_{g_{k}^{\prime}}+k \nu_{k}\right)^{m+r-1}}{(m+r-1) !}-\frac{V_{k}}{N_{k}} \int_{\mathbb{P} E^{*}}\left\langle t_{i}, t_{j}\right\rangle_{g_{k} \otimes \sigma_{k}^{\otimes k}} \frac{\left(\omega_{g_{k}}+k \nu_{k}\right)^{m+r-1}}{(m+r-1) !} \\
& =\frac{V_{k}}{N_{k}} \int_{\mathbb{P} E^{*}}\left\langle t_{i}, t_{j}\right\rangle_{g_{k} \otimes \sigma_{k}^{\otimes k}}\left(f_{k}\left(1+\epsilon_{k}\right)^{-1}-1\right) \frac{\left(\omega_{g_{k}}+k \nu_{k}\right)^{m+r-1}}{(m+r-1) !}
\end{aligned}
$$

where $\left(\omega_{g_{k}^{\prime}}+k \nu_{k}\right)^{m+r-1}=f_{k}\left(\omega_{g_{k}}+k \nu_{k}\right)^{m+r-1}$. By a unitary change of basis, we may assume without loss of generality that the matrix $M^{(k)}$ 
is diagonal. Thus

$$
\left\|M^{(k)}\right\|_{\text {op }} \leq \frac{V_{k}}{N_{k}}\left\|f_{k}\left(1+\epsilon_{k}\right)^{-1}-1\right\|_{L^{\infty}}
$$

On the other hand,

$$
\begin{aligned}
\left\|\omega_{g_{k}^{\prime}}-\omega_{g_{k}}\right\|_{C^{0}\left(\omega_{0}\right)} & =\left\|\bar{\partial} \partial \log \left(1+\epsilon_{k}\right)\right\|_{C^{0}\left(\omega_{0}\right)} \leq\left\|\log \left(1+\epsilon_{k}\right)\right\|_{C^{2}\left(\omega_{0}\right)} \\
& \leq-\log \left(1-C\left\|\epsilon_{k}\right\|_{C^{2}\left(\omega_{0}\right)}\right)=O\left(k^{-q-1}\right) .
\end{aligned}
$$

Therefore,

$$
\begin{aligned}
\left\|f_{k}-1\right\|_{\infty}=\left|\frac{\omega_{g_{k}^{\prime}}^{m+r-1}-\omega_{g_{k}}^{m+r-1}}{\omega_{g_{k}}^{m+r-1}}\right| & =\left|\frac{\omega_{g_{k}^{\prime}}^{m+r-1}-\omega_{g_{k}}^{m+r-1}}{\omega_{0}^{m+r-1}} \frac{\omega_{0}^{m+r-1}}{\omega_{g_{k}}^{m+r-1}}\right| \\
& \leq C k^{-q-1}\left|\frac{\omega_{0}^{m+r-1}}{\omega_{g_{k}}^{m+r-1}}\right| .
\end{aligned}
$$

This implies that $\left\|f_{k}-1\right\|_{\infty} \leq C k^{-q-1}$, since $\left|\frac{\omega_{0}^{m+r-1}}{\omega_{g_{k}}^{m+r-1}}\right|$ is bounded.

Hence $\left\|f_{k}\left(1+\epsilon_{k}\right)^{-1}-1\right\| \leq C^{\prime} k^{-q-1}$. Therefore $\left\|M^{(k)}\right\|_{\text {op }}=O\left(k^{-q-1}\right)$. Prop. 6.1 implies that $\mathbb{P} E^{*}$ has no nontrivial holomorphic vector fields. On the other hand

$$
\left\|\log \left(g_{k} \otimes \sigma_{k}^{\otimes k}\right)-\log \left(\widehat{h_{H E}} \otimes \sigma_{\infty}^{\otimes k}\right)\right\|_{C^{a+2}\left(\widetilde{\omega}_{0}\right)}=O(1) .
$$

Therefore, applying [S, Theorem 4.6.] and (4.7) conclude the proof.

\section{REFERENCES}

[BBS] R. Berman, B. Berndtsson, J. Sjöstrand, A direct approach to Bergman kernel asymptotics for positive line bundles, arXiv:math/0506367v2

[C] D. Catlin, The Bergman kernel and a theorem of Tian, in Analysis and geometry in several complex variables (Katata,1997), 1-23, Birkhäuser, Boston, Boston, MA.

[D] S. K. Donaldson, Scalar curvature and projective embeddings. I, J. Differential Geom. 59 (2001), no. 3, 479-522.

[K] S. Kobayashi, Differential geometry of complex vector bundles. Publications of the Mathematical Society of Japan, 15.Kan Memorial Lectures, 5. Princeton University Press, Princeton,NJ; Iwanami Shoten, Tokyo, 1987. xii+305 pp. ISBN: 0-691-08467-X

$[\mathrm{Lu}] \quad$ Z. Lu, On the lower order terms of the asymptotic expansion of TianYau-Zelditch, Amer. J. Math. 122 (2000), no. 2, 235-273. MR1749048 (2002d:32034)

[L] H. Luo, Geometric criterion for Gieseker-Mumford stability of polarized manifolds, J. Differential Geom. 49 (1998), no. 3, 577-599.

[M] I. Morrison, Projective stability of ruled surfaces, Invent.Math.56 (1980), no. 3, 269-304.

[PS1] D. H. Phong and J. Sturm, Stability, energy functionals, and KählerEinstein metrics, Comm. Anal. Geom. 11 (2003), no. 3, 565-597. 
[S] R. Seyyedali, Balanced metrics and Chow stability of projective bundles over Kähler manifolds, Duke Math. J. Volume 153, Number 3 (2010), 573-605.

[UY] K. Uhlenbeck and S.-T. Yau, On the existence of Hermitian-Yang- Mills connections in stable vector bundles, Comm. Pure Appl. Math. 39 (1986), no. S, suppl., S257-S293.

[W] X. Wang, Canonical metrics on stable vector bundles. Comm.Anal. Geom. 13 (2005), no. 2, 253-285.

[Z] S. Zelditch, Szegö kernels and a theorem of Tian, Internat.Math. Res. Notices 1998, no. 6, 317-331.

[Zh] S. Zhang, Heights and reductions of semi-stable varieties, Compositio Math. 104 (1996), no. 1, 77-105.

UCI, Department of Mathematics

E-mail address: rseyyeda@math.uci.edu 